\title{
A Monte Carlo Parametric Sensitivity Analysis of Automobile Handling, Comfort, and Stability
}

\author{
Mohamed A. Hassan, ${ }^{1,2,3}$ Mohamed AA Abdelkareem (i), ${ }^{3,4}$ Gangfeng Tan $\left(\mathbb{D},{ }^{1,2}\right.$ \\ and M.M Moheyeldein ${ }^{3}$ \\ ${ }^{1}$ School of Automotive Engineering, Wuhan University of Technology, Wuhan 430070, China \\ ${ }^{2}$ Suizhou-WUT Industry Research Institute, Suizhou 441300, China \\ ${ }^{3}$ Automotive and Tractors Engineering Department, Faculty of Engineering, Minia University, El-Minia 61519, Egypt \\ ${ }^{4}$ Department of Mechanical Engineering, The Hong Kong Polytechnic University, Hong Kong, China
}

Correspondence should be addressed to Gangfeng Tan; auto_nova@whut.edu.cn

Received 10 October 2020; Revised 16 August 2021; Accepted 21 August 2021; Published 7 September 2021

Academic Editor: Marco Lepidi

Copyright ( $\odot 2021$ Mohamed A. Hassan et al. This is an open access article distributed under the Creative Commons Attribution License, which permits unrestricted use, distribution, and reproduction in any medium, provided the original work is properly cited.

\begin{abstract}
This paper investigates the bandwidth sensitivity of automobile handling, comfort, and stability based on Monte Carlo sensitivity simulations. Performed bandwidth sensitivity simulations include the effects of vehicle geometry and suspension parameters on lateral acceleration, roll angle, front/rear sideslip angles, and yaw rate angle, including both time- and frequency-domain sensitivity analyses. To replicate actual automobile responses, a full-vehicle roll-oriented suspension seven-degree-of-freedom (7-DOF) model was developed and implemented considering a 2-DOF planar two-track model with a nonlinear Pacejka tire model. During the Monte Carlo simulations, $10 \mathrm{~mm}$ and $20 \mathrm{~mm}$ amplitude sine-wave excitations were used for the left and right sides, respectively, and the frequency was uniformly sampled over the range of $0-30 \mathrm{~Hz}$. Simultaneously, each investigated vehicle parameter varied by $\pm 25 \%$ relative to the reference model parameters. These simulations illustrate the sensitivity of the lateral acceleration, roll angle, yaw angle, and sideslip angles to their parameter variations. The results confirm that the road excitation frequency, tire properties, vehicle geometry, and suspension parameters significantly influence the vehicular lateral and roll stabilities when considering the lower and higher peaks and the frequency bandwidths of the lateral and roll stabilities. Interestingly, the longitudinal location of the center of gravity and the tire properties can achieve more significant peak lateral stability responses, as represented by the front and rear sideslip angles and the frequency bandwidth, compared to the other vehicle parameters at high frequencies. Choosing the correct tire properties and vehicle geometry, as well as suspension characteristics, plays an essential role in increasing the vehicular lateral stability and the rollover threshold. Variations in the studied parameters allow for higher vehicular stability when a vehicle is driven on random road surfaces.
\end{abstract}

\section{Introduction}

Studies of vehicle dynamics can make fundamental contributions to the further development of the vehicle industry. Accordingly, several strategies have been investigated to enhance the ride and handling performances of vehicles. Handling dynamics deal with the lateral or transverse vehicle dynamics, which primarily refer to the vehicular handling stability, sideslip caused by lateral tire forces, and yaw and roll motions. Vehicular handling and stability are significantly affected by the inertial vehicle properties, including the sprung mass and the location of the center of gravity (C.G.), suspension characteristics, and driving conditions [1-5]. Tuned vehicle design parameters and suspension features can provide enhanced interactions between the tires and the road surface [6-8].

1.1. Literature Review. Several studies have investigated the effects of vehicle parameters on the lateral and handling instabilities. Whitehead et al. [2] studied the impact of the C.G. location on the lateral and roll instabilities when a vehicle 
is subjected to a fishhook maneuver. The correlation between the C.G. location and the rollover resistance was determined using a validated three-degree-of-freedom (3-DOF) model. In another study by Marimuthu et al. [9], the sensitivity of the effect of critical vehicle parameters on the roll behavior was investigated for sport utility vehicles (SUVs) using a validated nonlinear vehicle model. The investigated vehicle parameters included the C.G. height, track width, and wheelbase length during a fishhook steering maneuver. Many other scientific articles have examined the lateral stability and rollover thresholds of heavy trucks in terms of the design parameters and operational factors, including the load distribution, trailer spacing, payload density, and C.G. height $[10,11]$.

In recent years, researchers have focused on the role of suspension characteristics in vehicle dynamics. There are many suspension types, including springs, shock absorbers, and anti-roll bars; therefore, it is vital to understand their corresponding vehicular lateral stability and rollover resistance capabilities. Zhang et al. [12] developed a 9-DOF vehicle model to evaluate the vehicular rollover propensity. The vehicle lateral acceleration, roll angle, roll rate, and yaw rate were obtained for different suspension stiffnesses during a fishhook maneuver. The study extended to the influences of shock absorber damping and the anti-roll bar on the vehicular rollover resistance capability [7]. The rollover critical factor has also been evaluated with variations in the suspension characteristics using the fishhook and sine maneuvers. The results indicate the strong effects of suspension properties, such as the roll center height, roll steer, and roll camber on the vehicle dynamics. Hac $[13,14]$ investigated the effects of various suspension design parameters that tend to increase the jacking forces and the vehicle rollover propensity using simple roll-plane and full-vehicle models. Shim and Velusamy $[6,15]$ studied the vehicle dynamics while changing the suspension geometry, including the toe, camber, and roll center height for both the front and rear suspensions with variations in wheel travel. Several studies have investigated hydraulically interconnected suspension systems to enhance both the lateral and roll stabilities via suspension tuning [16-21].

To reduce lateral movement and ensure ride quality, a magnetorheological (MR) damper has been widely used to connect the bogie and the vehicle body in practically all rail vehicles [22]. The MR damper was designed to be fitted into the secondary lateral suspension system of railway vehicles to improve ride quality through nonlinear stiffness and damping. Simulation results of different strategies for semiactive controlled MR lateral dampers were compared with the conventional passive lateral damper while the vehicle was excited at different running speeds and track irregularities, including the effects of various braking phases on longitudinal train dynamics [23].

Tire characteristics can provide improved interactions between the tire and the road surface [24-27]. Tire characteristics are influenced by several factors, including the tire parameters (e.g., material, tire type, tire tread depth, and internal pressure), vehicle parameters (e.g., speed and camber angle), vertical load, and road surface conditions [28]. In terms of vehicle parameters, the cross-section and size of a tire significantly affect the tire adhesion coefficient. Similarly, the tire adhesion coefficient is significantly influenced by the tire parameters, where increasing the tire size and decreasing the aspect ratio leads to an increase in the tire adhesion coefficient [29].

Conversely, increasing the tire pressure reduces the adhesion coefficient of the tire by reducing the tire-road surface contact area. Meanwhile, decreasing the tire pressure increases the adhesion coefficient by increasing the contact patch [30]. In addition, the adhesion coefficient is greatly influenced by the tread pattern, which increases the adhesion capacity of the tire by removing the moisture between the tread and the actual contact area on the road surface [31]. Vehicle speed also has a significant effect on the adhesion coefficient, which gradually decreases as the speed increases $[28,32]$. Road surface conditions dominate the tire adhesion coefficient; these conditions are the most critical factors for the tire adhesion coefficient, and the adhesion of the tire is strongly affected by wet and snowy conditions [33, 34]. Wielenga [35] investigated the effect of the tire characteristics for different-sized tires, including the coefficients of cornering, camber stiffness, and peak lateral friction. The rollover resistance and lateral stability were examined under normal and extreme maneuvers. The effects of the tire characteristics on vehicular rollover and lateral stability were investigated using two tire types with different adhesions to evaluate the relationship between the rollover propensity and lateral stability [5]. The results confirmed that the adhesion capacities of tires significantly influence the lateral vehicular stability and rollover propensity.

Wang et al. [36] studied roll instability under various road excitations and steering wheel inputs. In Ref. [37], the correlation between the vehicular rollover and lateral stabilities and the impact of road excitations was considered to evaluate the overall vehicular rollover stability. The effects of steering wheel inputs with different amplitudes and frequencies on an untripped rollover were evaluated in Ref. [38]. The results confirmed that, during an extreme maneuver, steering frequencies ranging from $0.3 \mathrm{~Hz}$ to $0.8 \mathrm{~Hz}$ are critical for untripped rollovers. Joshi et al. [39] studied the effect of the steering input frequency on the transient handling dynamics of SUVs. Their results indicate that the yaw rate and lateral acceleration responses are significantly correlated with low-frequency steering inputs below $2 \mathrm{~Hz}$. The critical rollover factor was determined considering the steering frequency with different steering amplitudes and vehicle speeds in Ref. [7]. The result revealed that rollovers occur within a frequency range of $0.52-0.6 \mathrm{~Hz}$.

Recently, Monte Carlo simulations have been introduced to perform comprehensive parameter sensitivity analyses in several automobile applications. Taghavifar and Rakheja [40] presented a parametric sensitivity analysis of the potential of energy harvesting, considering a four-wheeled generic three-dimensional full-car using Monte Carlo simulations of different suspension characteristics, on-road classes, and driving conditions. In another study, Zhang et al. [41, 42] conducted a comparative investigation of both direct and indirect drive vibratory energy harvesters concerning regenerative shock absorbers to assess their energy 
harvesting abilities using Monte Carlo sensitivity simulations. Abdelkareem et al. [43] introduced a frequency sensitivity analysis based on Monte Carlo simulations to perform a comprehensive bandwidth analysis of harvestable power and suspension dynamics. Their sensitivity simulations included the damping rate variation, spring stiffness, tire stiffness, sprung mass, unsprung mass, and harmonic excitation amplitude. Their results demonstrated the effectiveness of Monte Carlo simulations when investigating vehicle dynamics, especially in the frequency domain.

1.2. Paper Contribution. Most of the conducted studies mainly addressed how the vehicle design parameters affect vehicular lateral and roll stability, focusing only on timedomain analysis, which does not precisely characterize the vehicle stability performance in terms of the frequency bandwidth. Thus, it is necessary to design the vehicle geometry and suspension parameters to improve vehicle stability adequately during low-frequency and high-frequency band scenarios. Frequency-based sensitivity simulations aim to identify the least and most influential parameters/variables to reveal correlations between the parameters and the system performance. In this sense, continued efforts are necessary to provide greater insight into the impacts of both low- and high-frequency road excitations on vehicular lateral stability and rollover propensity. Based on Monte Carlo simulations, the current study conducted a complete frequency-based parametric bandwidth sensitivity analysis associated with vehicle lateral and roll stability of a 9-DOF passenger vehicle model. The sensitivity simulations included inertial vehicle properties, suspension system characteristics, and harmonic excitation parameters. The lateral and transverse dynamics of the vehicle, which primarily refer to vehicular handling stability, front/rear tire sideslip caused by lateral tire forces, lateral acceleration, roll angle, and yaw rate, were investigated. Furthermore, the contribution of this paper also lies in identifying the parameters/variables that are either most or least important for vehicular lateral and rollover stabilities from a frequency-domain perspective. These frequency-based parametric bandwidth sensitivity analyses also highlight the bandwidth change in the peak amplitudes and resonant frequency bandwidths of the lateral stability and rollover propensity. Thus, complete frequency-based bandwidth sensitivity simulations would also provide insights into the on-resonance and off-resonance performance of the lateral stability and rollover propensity.

\section{Vehicle Modeling}

A 9-DOF nonlinear vehicle model is developed to describe the vertical and lateral dynamics, including sprung mass lateral and vertical motions, roll and pitch rotations of the sprung mass about the center of gravity axis, yaw rotation of the total vehicle mass, and unsprung mass bounce motions.
2.1. Ride Comfort Model. Real suspensions have nonlinear springs and dampers, whose features cannot be reduced to a single number like the linear case. However, suspensions with linear behavior are an excellent introduction to the study of the ride and vehicle stability in the literature due to modeling simplicity $[12,44]$. Inspired by this, our paper ignored the nonlinearity of suspension components. The ride comfort model consists of 7-degree of freedom, consisting of the vehicle body bounce, pitch, roll, and four wheels vertical motions, as shown in Figure 1(a). It is worth mentioning that the lateral acceleration is brought into the vehicle body's roll motion as illustrated in Figure 1(b). Moreover, the suspension model provides the tire model's dynamic tire load, which plays a vital role. It is the major link between the vertical and lateral forces exerted on the road, which affects the vehicle's lateral dynamics. The suspension and roll motion models are subjected to sine-wave road irregularities, as illustrated in Section 3.

The equation of motion of the suspension system and roll motion can be developed as follows.

The vertical vibration and pitch motion of the sprung mass is expressed as

$$
M_{b} \ddot{z}_{b}=F_{s f r}+F_{s f l}+F_{s r r}+F_{s r l},
$$

where the forces $F_{s r r}, F_{s r l}, F_{s f r}$, and $F_{s f l}$ are the suspension forces for each tire.

$$
I_{y} \ddot{\theta}_{b}=\left(F_{s r r}+F_{s r l}\right) \cdot l_{r}-\left(F_{s f r}+F_{s f l}\right) \cdot l_{f} .
$$

The vertical vibration of unsprung masses is represented as follows:

$$
\begin{aligned}
m_{u f r} \ddot{z}_{u f r} & =k_{t f r}\left(z_{R f r}-z_{u f r}\right)-F_{s f r}, \\
m_{u f l} \ddot{z}_{u f l} & =k_{t f l}\left(z_{R f l}-z_{u f l}\right)-F_{s f l}, \\
m_{u r r} \ddot{z}_{u r r} & =k_{t r r}\left(z_{R r r}-z_{u r r}\right)-F_{s f r r}, \\
m_{u r r} \ddot{z}_{u r l} & =k_{t r l}\left(z_{R r l}-z_{u r l}\right)-F_{s f r l} .
\end{aligned}
$$

Then, the vehicle body roll motion can be expressed as follows:

$$
\begin{aligned}
I_{x} \ddot{\phi}= & \left(F_{s r r}+F_{s r l}\right) \cdot \frac{b_{r}}{2}-\left(F_{s f r}+F_{s f l}\right) \cdot \frac{b_{f}}{2}+M_{b} a_{y} h_{c} \cos \phi \\
& +M_{b} g h_{c} \sin \phi .
\end{aligned}
$$

The dynamic suspension forces mentioned in the previous equations can be derived as follows:

$$
\begin{aligned}
& F_{s f r}=k_{s f r}\left(z_{u f r}-z_{b f r}\right)+c_{s f r}\left(z_{u f r}-z_{b f r}\right), \\
& F_{s f l}=k_{s f l}\left(z_{u f l}-z_{b f l}\right)+c_{s f l}\left(z_{u f r}-z_{b f l}\right), \\
& F_{s r r}=k_{s r r}\left(z_{u r r}-z_{b r r}\right)+c_{s r r}\left(z_{u r r}-z_{b r r}\right), \\
& F_{s r l}=k_{s r l}\left(z_{u r l}-z_{b r l}\right)+c_{s r l}\left(z_{u r r}-z_{b r l}\right) .
\end{aligned}
$$

According to geometry relations, body deflections can be calculated as 


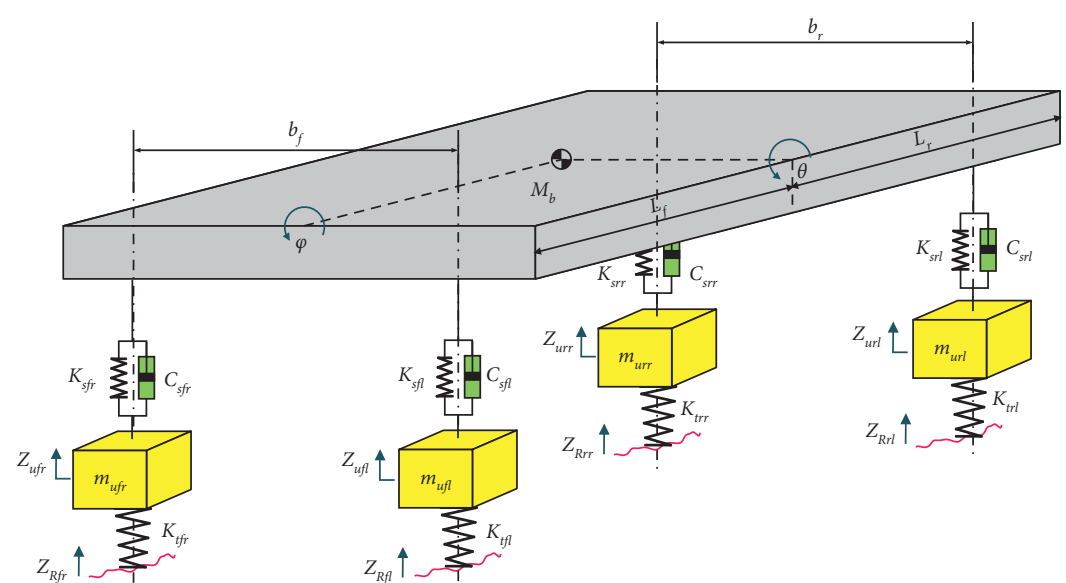

(a)

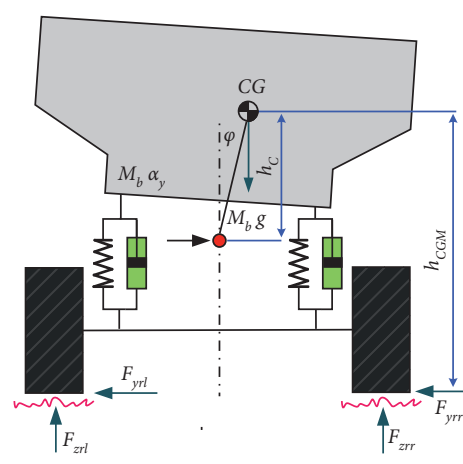

(b)

Figure 1: A seven-degree-of-freedom full-car model.

$$
\begin{gathered}
z_{b f r}=z_{b}-l_{f} \theta-\frac{b_{f} \phi}{2} \cdot z_{b f l}=z_{b}-l_{f} \theta+\frac{b_{f} \phi}{2}, \\
z_{b r r}=z_{b}+l_{r} \theta+\frac{b_{r} \phi}{2} \cdot z_{b f l}=z_{b}+l_{r} \theta-\frac{b_{r} \phi}{2} .
\end{gathered}
$$

Vertical tire forces at each tire, including both static and dynamic tire load, can be determined as the following equation:

$$
\begin{gathered}
F_{z f r}=\frac{M_{b} l_{r}}{l_{r}+l_{f}} g+m_{u f r} g+k_{t f r}\left(z_{R f r}-z_{u f r}\right), \\
F_{z f r}=\frac{M_{b} l_{r}}{l_{r}+l_{f}} g+m_{u f l} g+k_{t f l}\left(z_{R f l}-z_{u f l}\right), \\
F_{z r r}=\frac{M_{b} l_{f}}{l_{r}+l_{f}} g+m_{u r r} g+k_{t r r}\left(z_{R r r}-z_{u r r}\right), \\
F_{z r l}=\frac{M_{b} l_{f}}{l_{r}+l_{f}} g+m_{u r l} g+k_{t r r}\left(z_{R r l}-z_{u r l}\right) .
\end{gathered}
$$

In many studies, road input is used as a force directly coupled with the tire model resulting from the road profile amplitude and the tire's stiffness and damping [36, 37, 43]. Inspired by this, we have also considered the road input excitation (road amplitude seen in equation (7)) into our case study as a force resulting from the tire stiffness and amplitude difference of the road amplitude and unsprung mass displacement.

2.2. Tire Model. In the coupled lateral and vertical model, the vehicle dynamics are caused by the forces and moments generated between tires and road surface. It is known that the calculation of the tire-road friction force represents a great challenge due to its highly nonlinear dependence on many uncontrollable environmental characteristics.
Therefore, many empirical static tire models, such as the LuGre model, Burckhardt/Kiencke model, and the Dugoff model, were developed to describe nonlinear tire characteristics. To simulate a real vehicular response, Pacejka Magic Tire Formula (MTF) model is adopted to represent the response of nonlinear tire forces instead of linear cornering stiffness. In this Paper, the MTF models are easy to implement and have been widely proved to be accurate in many vehicle dynamics publications [45]. This tire model was selected because of its ability to accurately simulate tire behavior in high lateral acceleration and large tire defamation cases. Two tire types with different adhesive coefficients were chosen to address the conflict between the vehicle rollover propensity and lateral stability. For pure sideslip conditions, the nonlinear M.F. equations can be described as

$$
\begin{aligned}
F_{y i} & =D \sin \left(C \tan ^{-1}(B \phi)\right)+S_{v}, \\
\phi & =(1-E)\left(\alpha_{i}+S_{h}\right)+\frac{E}{B} \tan ^{-1}\left(B\left(\alpha_{i}+S_{h}\right)\right),
\end{aligned}
$$

where the unit of tire lateral force $F_{y i}$ and vertical tire force $F_{z i}$ is $\mathrm{kN}$, the unit of tire sideslip angle $\alpha_{i}$ is radian, and the factors $B, C, D, E, S_{h}$, and $S_{v}$ are stiffness factor, shape factor, peak value, curvature factor, horizontal shift, and vertical shift, respectively. The subindex (i) stands for tire left front, left rear, right front, and right rear.

2.3. Steering Model. The steering model utilized in this paper is a 2-DOFs model, as shown in Figure 2. These 2-DOFs donate for the lateral and yaw motions of the vehicle. During the cornering conditions, the lateral force is applied to the C.G. of the vehicle body and thus induced the rollover. The equations of the steering model are given in equations (9) and (10). The yaw equation of motion is governed in equation (9), while the lateral motion equation is given in equation (10). 


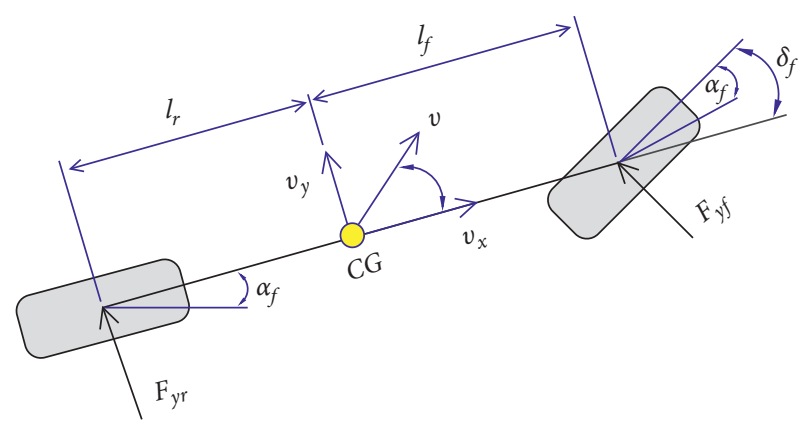

Figure 2: Bicycle steering model.

$$
\begin{gathered}
I_{z} \dot{r}=\left(F_{y f r} \cos \delta_{f}+F_{y f l} \cos \delta_{f}\right) l_{f}-\left(F_{y f r}+F_{y f l}\right) l_{r}, \\
M_{\text {tot }}\left(\dot{v}_{y}+v_{x} r\right)=\left(F_{y f r} \cos \delta_{f}+F_{y f l} \cos \delta_{f}\right)-\left(F_{y f r}+F_{y f l}\right) .
\end{gathered}
$$

The slip angles of the front axle $\alpha_{f}$ and rear axle $\alpha_{r}$ can be defined in terms of the variables $v_{y}$ and $r$ and using the usually small-angle assumptions.

$$
\begin{gathered}
\alpha_{f}=\delta_{f}-\frac{v_{y}+l_{f} r}{v_{x}}, \\
\alpha_{r}=-\frac{v_{y}-l_{b} r}{v_{x}} .
\end{gathered}
$$

The lateral acceleration is

$$
a_{y}=\dot{v}_{y}+v_{x} r
$$

where $v_{x}$ is the straightforward vehicle speed, $v_{y}$ is the lateral vehicle speed, $r$ is the yaw rate, $M_{\text {tot }}$ is the vehicle mass including sprung and unsprung masses, and $\delta_{f}$ denotes the front wheel steering angle.

\section{Parametrical Sensitivity Simulation Setup}

From parameter sampling based on Monte Carlo principles to results analysis, a comprehensive workflow diagram and modeling assembly are shown in Figure 3. To begin with, the Mote-Carlo technique was used to sample parameters. The input forces are then completed using the road excitation and generated parameters. Following that, the Pacejika Magic tire model is used to bridge the gap between the vehicle handling model and the road input. The Magic Formula (MF) tire model is used because it is simple to use and has been demonstrated to be accurate in several vehicle dynamics simulations. The dynamic tire load (DTL) plays a vital role as it is the primary link between the vertical and lateral forces exerted on the road. The lateral acceleration, which is the principal cause of the vehicle's body roll motion, is calculated using the steering and yawing motion model. The steering model uses the lateral force provided by the tire model to predict lateral acceleration. The MATLAB/Simulink was used to run the vehicle model's equation of motion. The bandwidth sensitivity simulation results were recorded in RMS and Peak values to show the frequency and amplitude bandwidth sensitivity to the proposed parametric analysis. The lateral vehicle dynamic and roll stability responses were normalized by their reference values recorded using the vehicle's nominal values. Fundamentally, Monte Carlo simulations analyze uncertainties in a probabilistic manner, in which the values of the specified parameters are sampled randomly during the simulation run depending on the input probability distributions [42, 43]. Uniform, normal, and triangular probability distributions are examples of input probability distributions. The Monte Carlo sensitivity approach, in particular, allows for the simulation of real driving circumstances in frequency domains [40].

The conducted simulations started with a time-domain sensitivity change analysis of the automobile stability and rollover responses (lateral acceleration, roll angle, yaw angle, and sideslip angles) with respect to a variation ratio of $\pm 25 \%$ of model parameters. During the simulations, a sine-wave signal was used with amplitudes of 10 and $20 \mathrm{~mm}$ for the left and right sides, respectively, with a frequency range of 0-30 Hz. During each simulation round for a single parameter sensitivity plot, 1600 sample points are generated for randomization of each parameter with respect to the randomly sampled frequency. The goal of these sensitivity simulations was to show how sensitive the 9-DOFs car suspension model's lateral acceleration, roll angle, yaw angle, and sideslip angles are to parameter modifications in terms of the frequency domain.

Sensitivity simulations are conducted following the step-by-step simulation flowchart, as seen in Figure 4. Firstly, the reference values of the model parameters are used for obtaining the reference trends of the investigated model outputs (lateral acceleration, roll angle, and sideslip angles). Thereafter, the Monte Carlo simulations are performed after setting and initializing the Monte Carlo algorithm parameters, including the sampling parameters of both the excitation frequency and the picked parameter. During the proposed Monte Carlo trials, the input excitation frequency is uniformly sampled in the range of $0-30 \mathrm{~Hz}$ to include the well-known modal resonant frequencies of the automobile stability and handling criteria. On the other hand, the investigated parameters are uniformly distributed with $\pm 25 \%$ based on their reference values. During the planned simulations, each investigated parameter is sampled based on the uniform probability function with 40 samples per parameter, in which each investigated response has 1600 simulation results to generate the plotted sensitivity figures. The selected parameters of the 7-DOFs vehicle suspension and roll model include suspension stiffnesses $\left(K_{\text {sf }}\right.$ and $\left.K_{\text {sr }}\right)$, damping coefficients $\left(C_{\text {sf }}\right.$ and $\left.C_{\mathrm{sr}}\right)$, wheelbase $(L)$, wheel track $(B)$, and center of gravity height $\left(h_{\mathrm{cg}}\right)$. Variation of parameters could be physically achieved either by changes in materials, manufacturing processes, or vehicle type. The main reason for this is that vehicle initial parameters and suspension characteristics are the most effective parameters for determining lateral stability and rollover behavior of the vehicle and roll angle threshold, as mentioned in the paper's literature section. Secondly, the majority of time- 


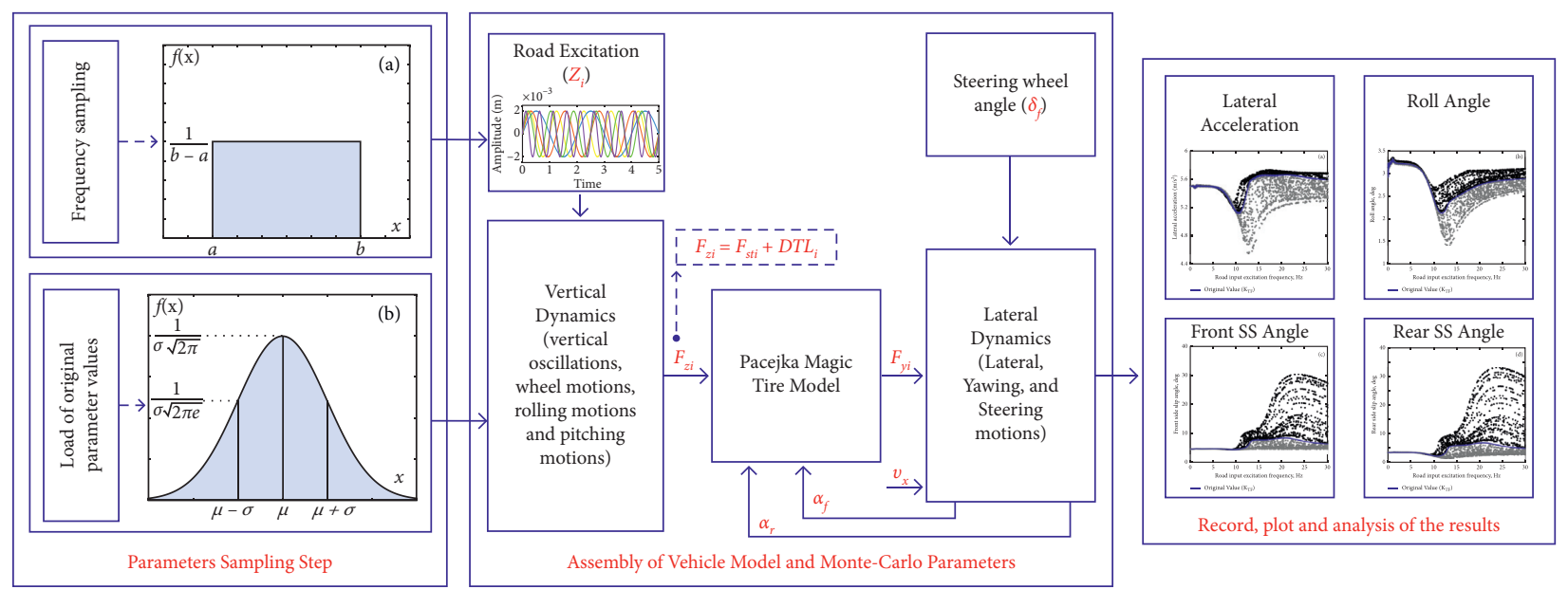

Figure 3: Complete workflow diagram and modeling assembly from parameters sampling to results analysis.

domain parametric research has concentrated on these variables, which have a strong relationship with lateral and roll stability. In this regard, the aforementioned parameters, which are the most influential, were also considered in our frequency-domain parametric sensitivity simulations. The concluded results of these parametrical bandwidth sensitivity simulations can also guide the control approach to enhance automobile handling, stability, and rollover responses. In Figure 4, the detailed schematic flow diagram of the conducted bandwidth parametrical sensitivity simulation is clarified. The simulation procedures can be highlighted as follows:

(1) In the first simulation step, the reference parameters are loaded, and after that, the typical trends of the lateral acceleration, roll angle, front sideslip angle, and rear sideslip angle are calculated with respect to the excitation frequency in the range of $0-30 \mathrm{~Hz}$.

(2) In the second phase, the Monte Carlo sampling parameters are initialized with respect to the uniform probability distribution with a sampling of 40 samples for each selected parameter. In this manner, the excitation frequency was randomly sampled in the range of $0-30 \mathrm{~Hz}$ and a sampling of 40 samples. The remaining parameters were randomly sampled with a variation of $\pm 25 \%$ based on the reference parameter's value and 40 samples sampling.

(3) In this stage, during each simulation round, the lateral acceleration, roll angle, front sideslip angle, and rear sideslip angle indexes are calculated and stored to perform the parametric sensitivity analysis in the frequency domain considering the Monte Carlo simulations.

(4) In the final step, the implemented algorithm picks up a new parameter to investigate its correlation sensitivity versus the defined automobile stability and rollover responses until all defined model parameters are investigated.

\section{Results and Discussion}

Speaking about the results' correctness and model validity, the primary simulation results and trends reported in Figure 5 normally appeared and are in agreement with similar results in Refs. [2, 12, 46]. Figure 5 depicts the vehicle's lateral acceleration, roll angle, roll rate, and yaw rate during the fishhook maneuver. These initial findings are essentially identical and in good agreement with those found in the literature, indicating that the models used are valid for further study and simulation.

The time-domain-based correlation sensitivity results for the automobile handling and stability responses, including lateral acceleration, roll angle, yaw rate, and front and rear sideslip angles, are presented in Figure 6 with respect to $\pm 25 \%$ variations in each of the chosen parameters based on the reference parameter value. It is apparent from Figure 6 that the lateral acceleration of a vehicle is strongly correlated with the wheelbase length of the vehicle with a reverse proportionality, i.e., an increase in the wheelbase length reduces the lateral acceleration and thereby enhances the stability of the vehicle. When the wheelbase length decreases by $25 \%$, the lateral acceleration increases by nearly $2 \%$, while a $5 \%$ decrease in the lateral acceleration corresponds to a $25 \%$ increase in the wheelbase length. It can also be concluded from Figure 6(a) that the lateral acceleration presents similar correlations with both the wheel track length and the C.G. height. These sensitivity correlations and outputs are in good agreement with the results published in Ref. [3].

Conversely, the lateral acceleration responded weakly to $\pm 25 \%$ variations in the suspension stiffness [12], damping rate, and tire stiffness, as revealed in Figure 6(a). An analysis of how sensitive the roll angle is to the model parameters is 


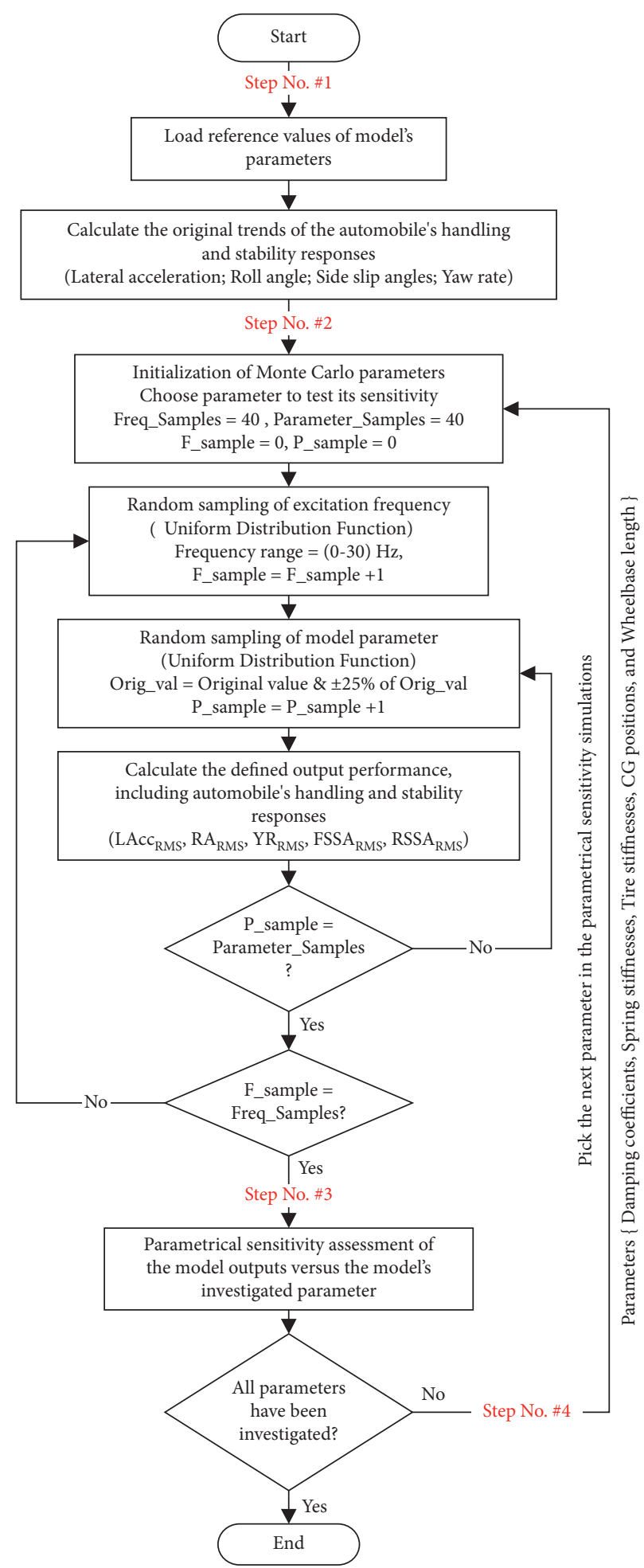

FIGURE 4: Step-by-step simulation flowchart of the conducted sensitivity simulations (LAccRMS: lateral acceleration RMS; RARMS: role angle RMS; YRRMS: yaw rate RMS; FSSARMS: front sideslip angle RMS; RSSARMS: rear sideslip angle RMS).

given in Figure 6(b). In Figure 6(b), the vehicle roll angle is strongly correlated first to the wheel track and second to the C.G. height of the vehicle. The roll angle response presents a slight increase with $+25 \%$ variations in the suspension stiffnesses and damping rates. Conversely, the results show a weak correlation and sensitivity to the variation in the vehicle wheelbase length. In other words, when the vehicle wheel track length decreases by $25 \%$, the roll angle increases by nearly $88 \%$, while a $38 \%$ decrease in the roll angle is observed, corresponding to a $25 \%$ increase in the wheel track length. Referring to Figures 6(c)-6(e), an analysis of how sensitive the sideslip and yaw angles are to the model parameters indicates 

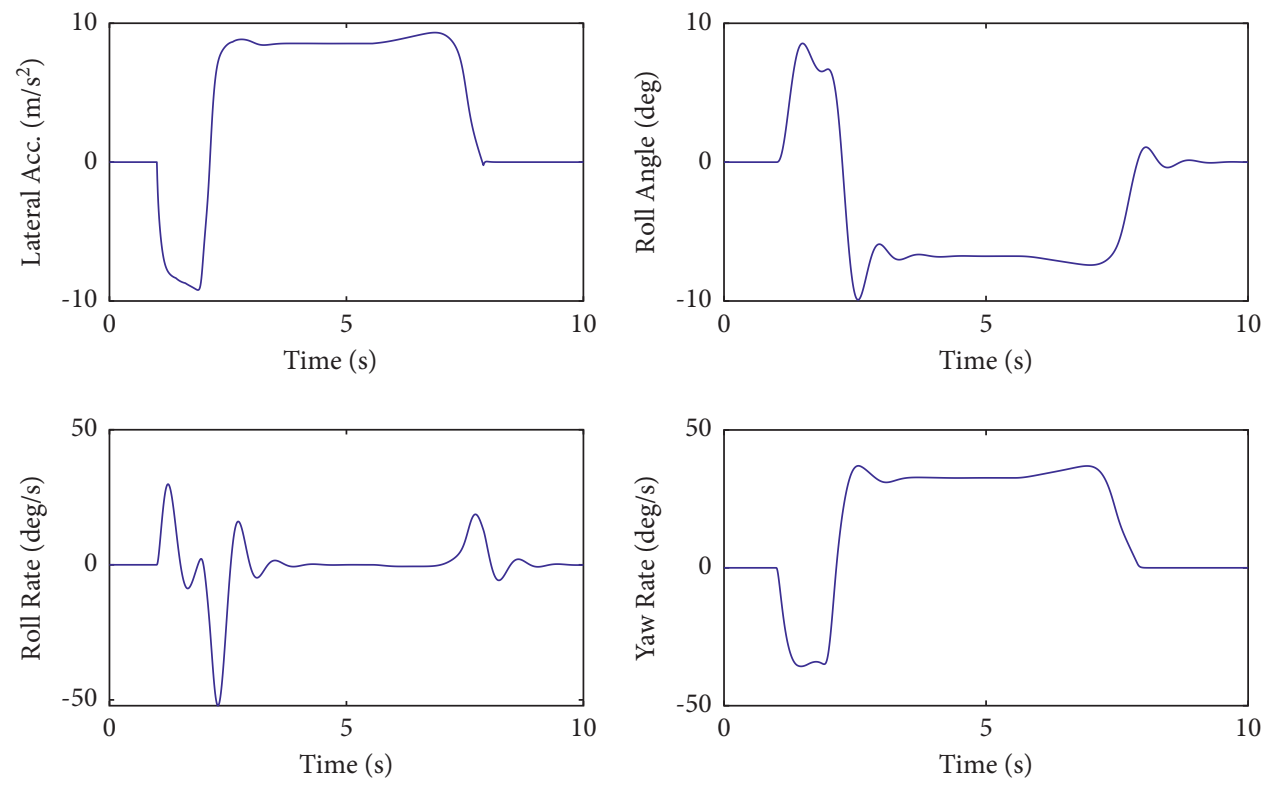

FIGURE 5: Primary results of the assembled 9-DOF nonlinear vehicle model under Fishhook Maneuver.

that both the sideslip and yaw angles are robustly correlated with the vehicle wheelbase length. Conversely, the results indicate that the damping coefficients and suspension stiffnesses hardly influence the sideslip and yaw angles. When the vehicle wheelbase length decreases by $25 \%$, the front sideslip, rear sideslip, and yaw angles increase by nearly 30,18 , and $3 \%$, respectively. In comparison, these responses decrease by 28 , 19 , and $6 \%$, respectively, for a $25 \%$ increase in the vehicle wheelbase length. These results are similar to Ref. [3], who also found that vehicles with narrower wheel track widths have an increased rollover propensity.

4.1. Front Damping Coefficient. Figure 5 shows the Monte Carlo parametric sensitivity results for the lateral acceleration, roll angle, and sideslip angles with respect to the front damping rate parameter. The front damping coefficient is varied using its nominal value and a standard deviation of $25 \%$. Changing the front damping results in significant variations in the abovementioned responses. However, the modal resonant frequencies are hardly changed. In Figures 7(a) and 7(b), in terms of the peak amplitude, the lateral acceleration and roll angle responded remarkedly to the tuning of the front suspension damping at a modal-resonant frequency of approximately $11 \mathrm{~Hz}$. The resonance of the front and rear sideslip angles is located at nearly $20 \mathrm{~Hz}$, as shown in Figures 7(c) and 7(d).

In Figure 7(a), it is inferred that the peak value of the lateral acceleration drops from $5.11 \mathrm{~m} / \mathrm{s}^{2}$ at the reference damping value to $4.64 \mathrm{~m} / \mathrm{s}^{2}$, showing a reduction ratio of $10 \%$ when the front damping rate decreases by $25 \%$. The peak value decreases from $2.1^{\circ}$ at the nominal damping to $1.7^{\circ}$ in the roll angle, resulting in a reduction ratio of $20 \%$, as seen in Figure 7(b). The sideslip angles present approximately similar sensitivities with respect to the front damping factor for the other variations. It can also be seen that vehicles with increased front damping present lower lateral and roll stabilities during a fishhook maneuver than a standard vehicle.

4.2. Rear Damping Coefficient. The sensitivity results for the automobile handling and stability responses (lateral acceleration, roll angle, and front and rear sideslip angles) with respect to the rear suspension damping coefficient are shown in Figure 8. In terms of the lateral acceleration response in Figure 8(a), the lateral acceleration root mean square (RMS) peak magnitude drops by nearly $2 \%$, corresponding to a $25 \%$ increase in the rear damping, which infers that the lateral acceleration responds slightly to the variation in the rear damping coefficient. Referring to Figures $8(\mathrm{c})$ and $8(\mathrm{~d})$, the variation in the rear suspension damping rate $( \pm 25 \%$ of the reference value) indicates higher sensitivity changes in the magnitudes of the front and rear sideslip angles. Conversely, the RMS trends of both the lateral acceleration and the roll angle present lower sensitivities than the sideslip angles, as indicated in Figures 8(a) and 8(b). When the rear damping coefficient drops to $25 \%$ of its nominal value, the peak amplitudes of the front and rear sideslip angles increase by $20 \%$ and $27 \%$, respectively, without a significant shift in their resonant frequencies.

However, when the rear damping parameter increases by $25 \%$, the sideslip angles of the front and rear tires decrease by $19 \%$ and $28 \%$, respectively. Therefore, the sideslip angles are remarkedly correlated with the suspension damping parameter and are inversely proportional to the damping coefficient variation. The modal resonant frequencies of the front and rear sideslip angles shift forward somewhat by almost 1.3 and $0.7 \mathrm{~Hz}$, respectively, as seen in Figures 8(c) and 8 (d), with a $\pm 25 \%$ variation in the rear damping rate. 

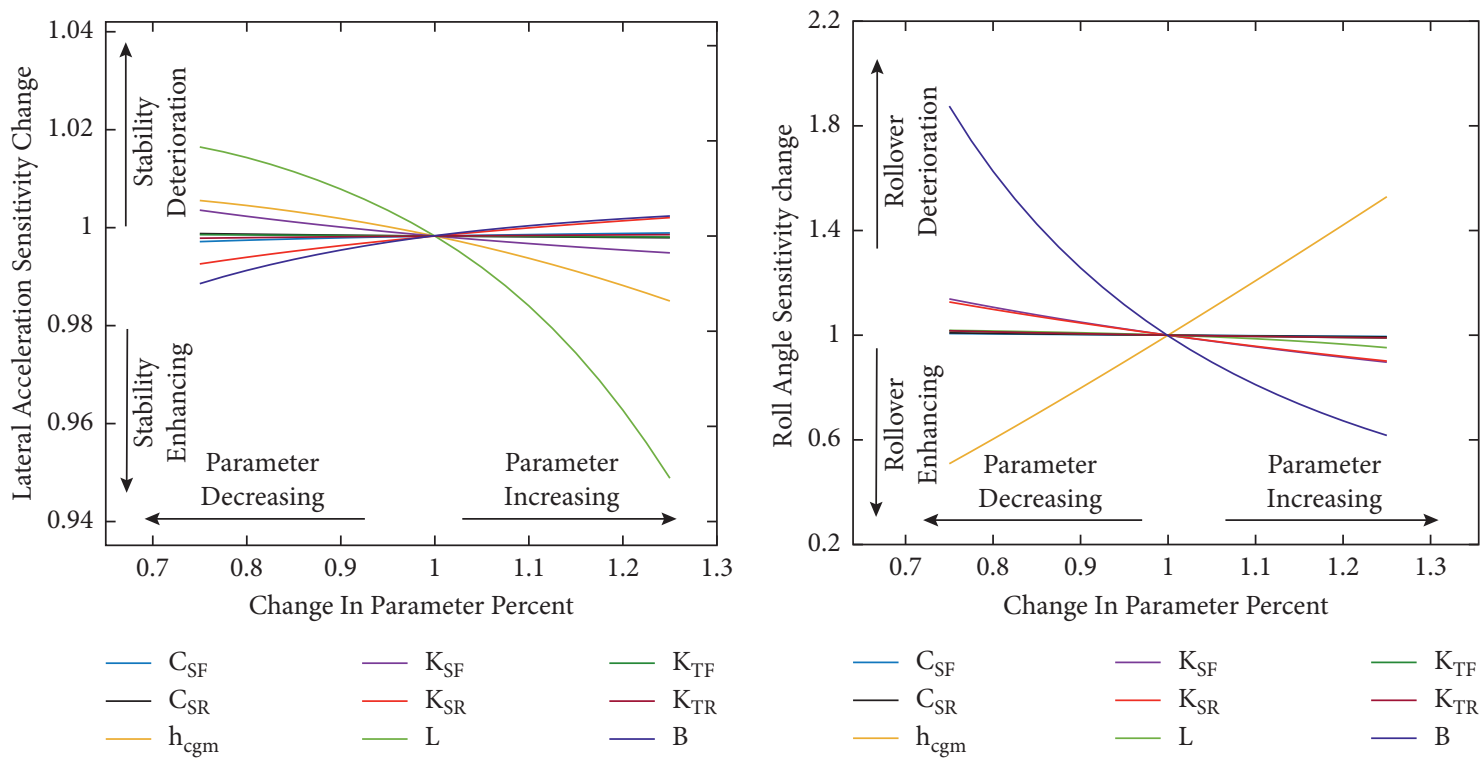

(a)
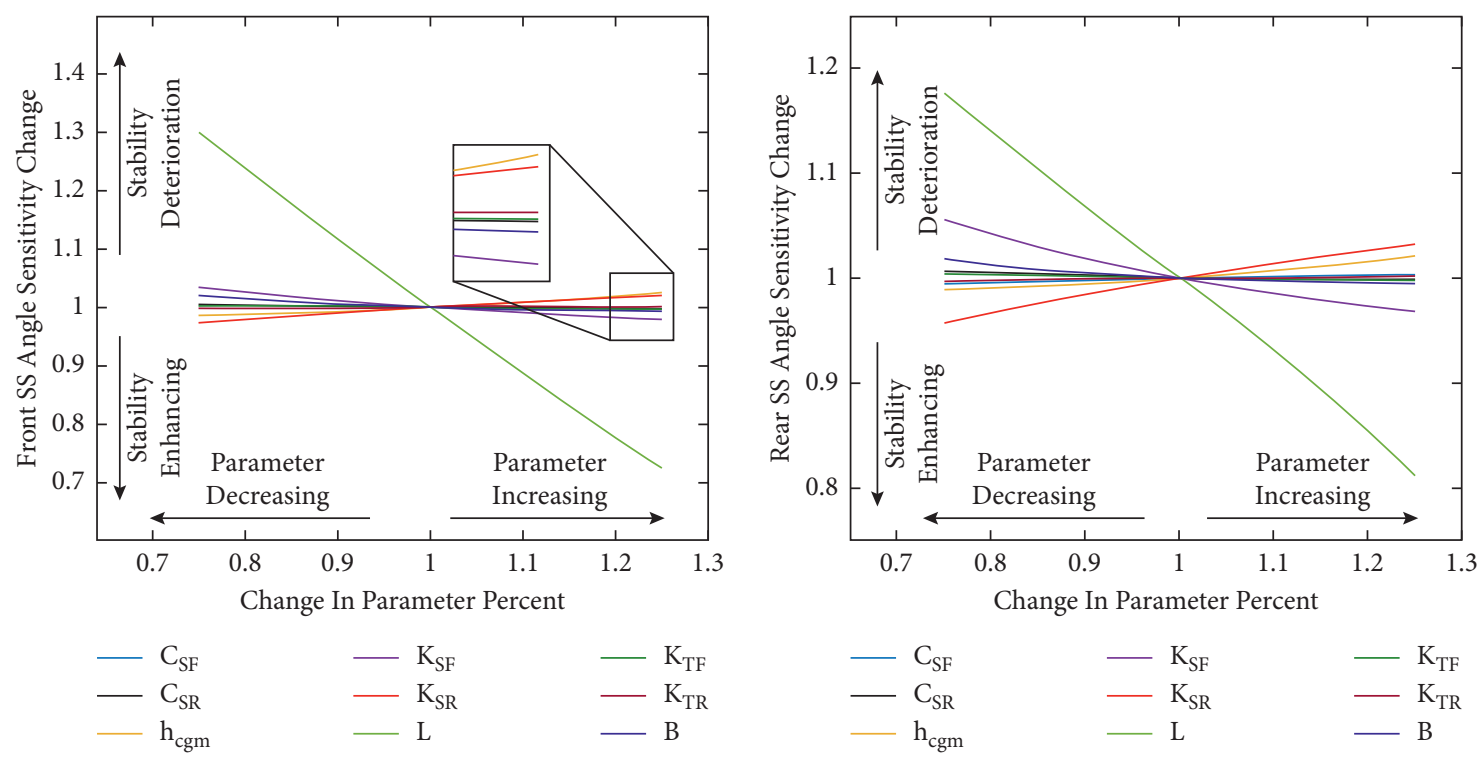

(c)

(d)

Figure 6: Continued. 


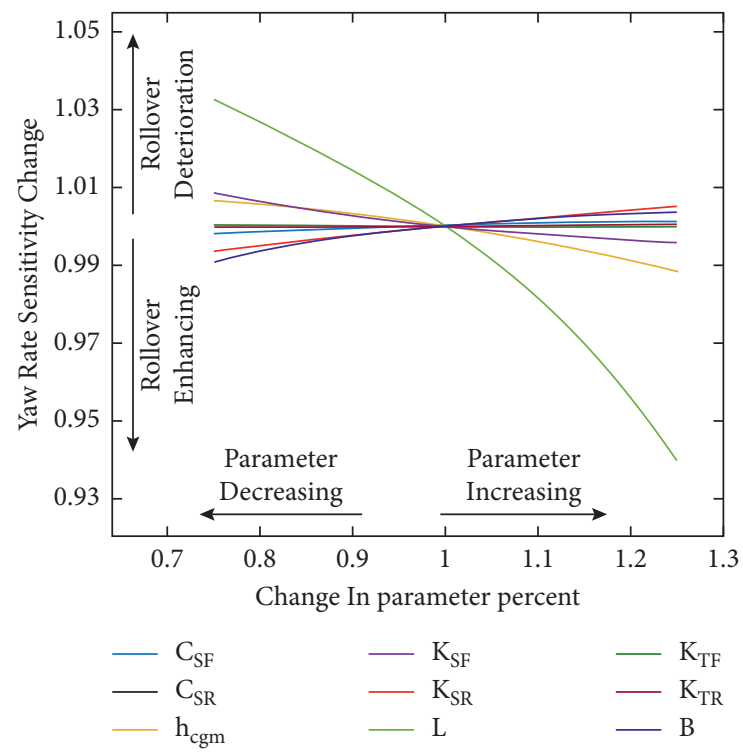

(e)

Figure 6: Simulation results for the automobile handling and stability response sensitivities with respect to the time-domain results. (a) Lateral acceleration response; (b) roll angle response; (c) front sideslip angle response; (d) rear sideslip angle response; (e) yaw rate angle response.

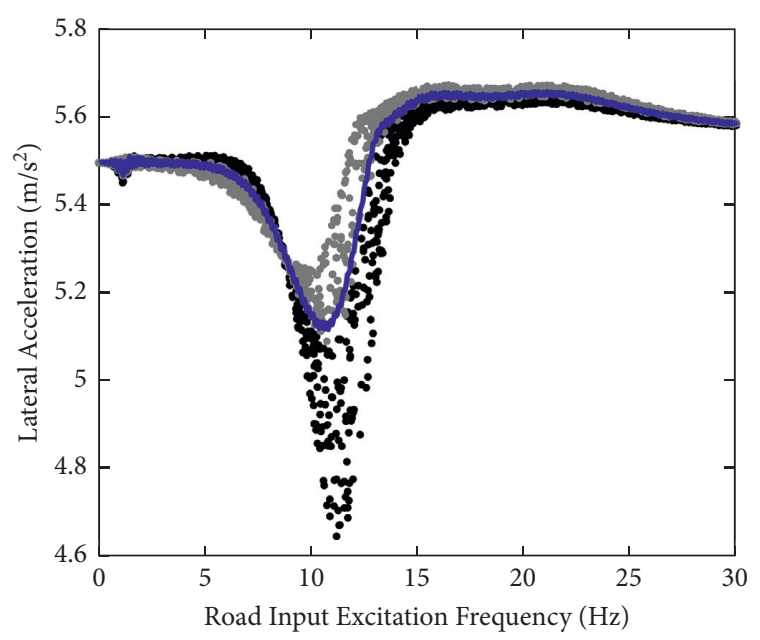

- Original Value $\left(\mathrm{C}_{\mathrm{F}}\right)$

- $-25 \%$ of Ref. value

- $+25 \%$ of Ref. value

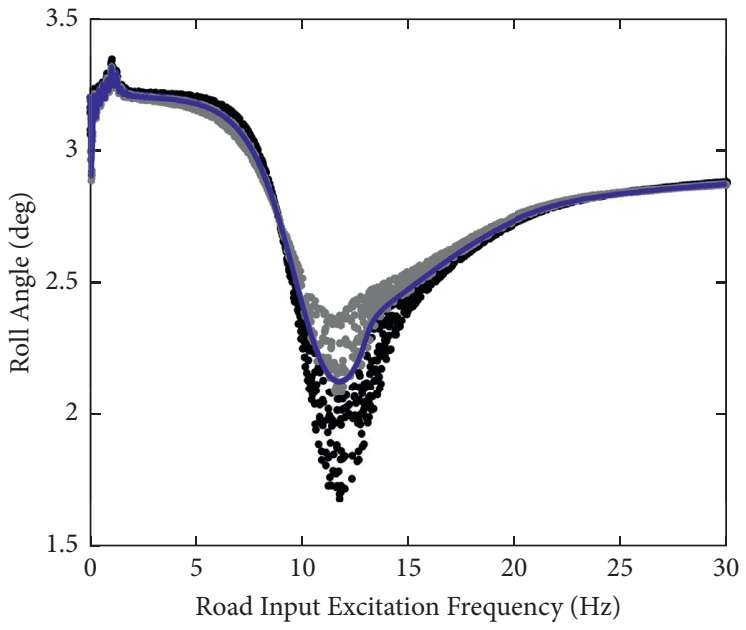

_ Original Value $\left(\mathrm{C}_{\mathrm{F}}\right)$

- $-25 \%$ of Ref. value

- $+25 \%$ of Ref. value

(a)

FIgURE 7: Continued. 


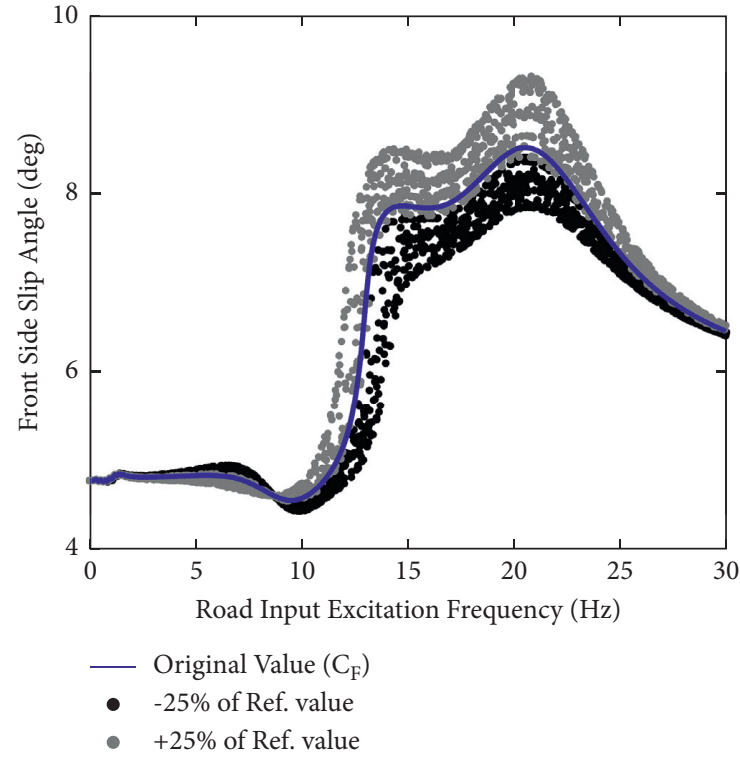

(c)

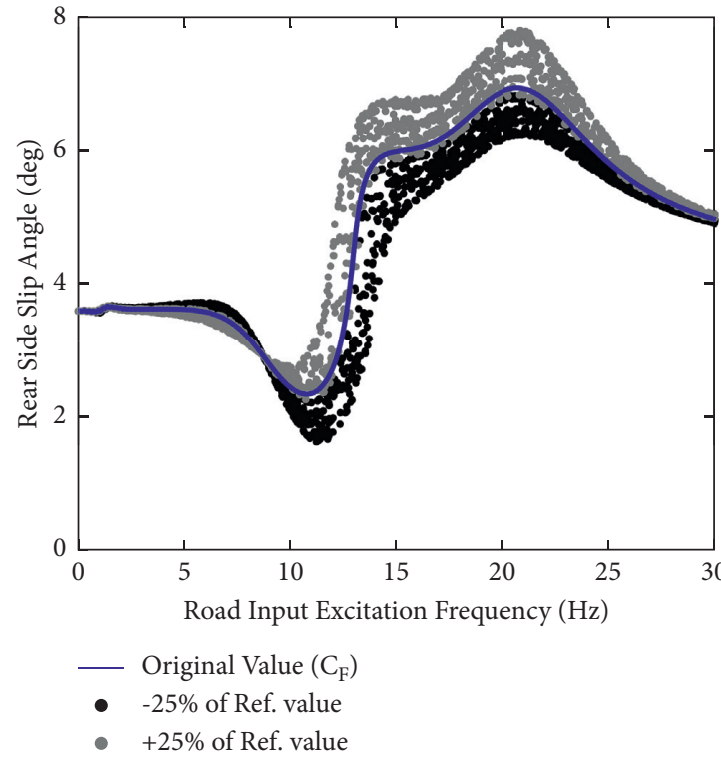

(d)

Figure 7: Sensitivity simulation results for the automobile handling and stability responses with respect to the front damping coefficient parameter. (a) Lateral acceleration response; (b) roll angle response; (c) front sideslip angle response; (d) rear sideslip angle.

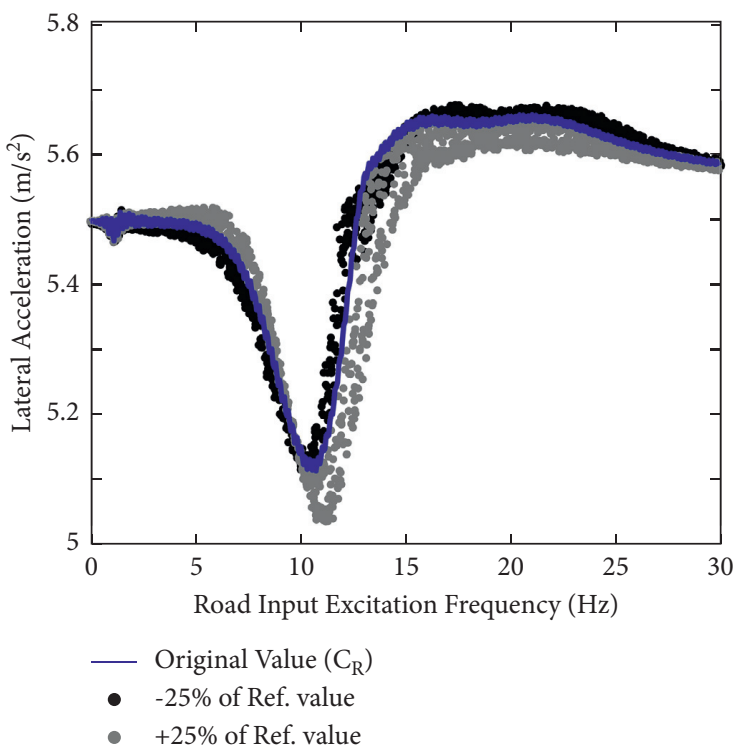

(a)

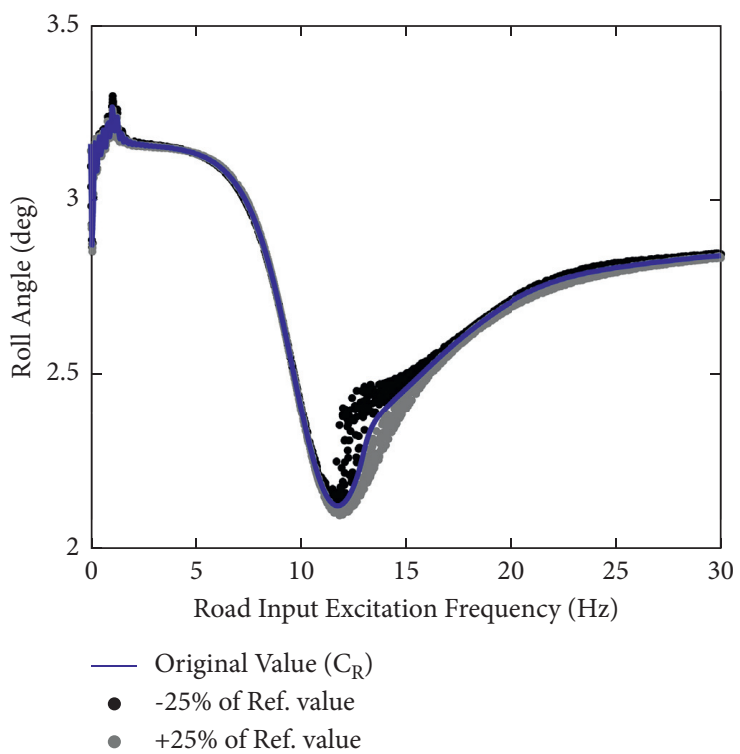

(b)

Figure 8: Continued. 


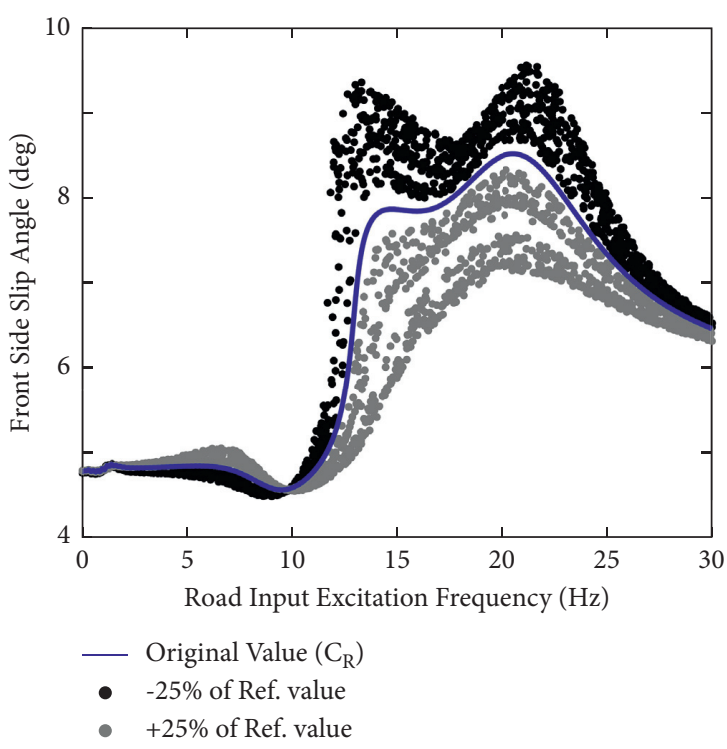

(c)

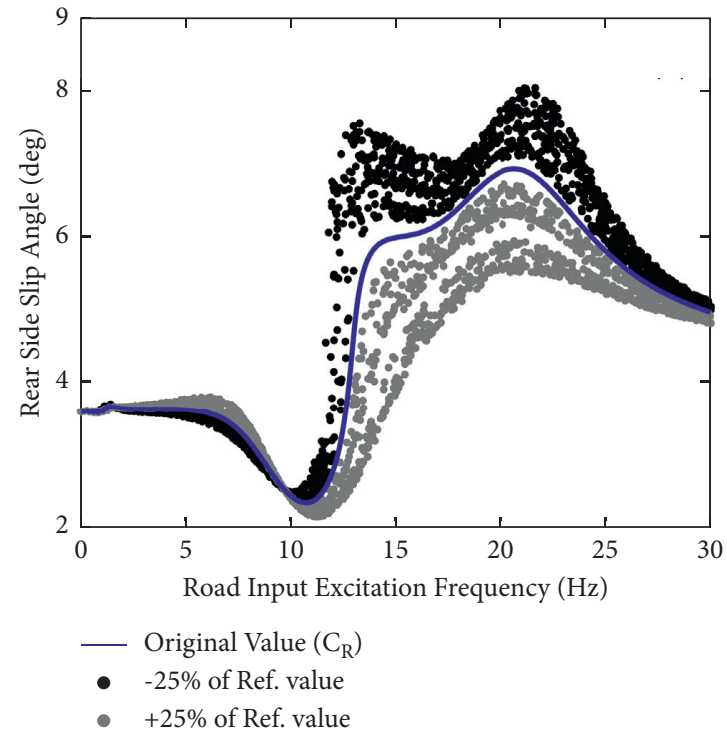

(d)

FIGURE 8: Sensitivity simulation results for the automobile handling and stability responses with respect to the rear damping coefficient parameter. (a) Lateral acceleration response; (b) roll angle response; (c) front sideslip angle response; (d) rear sideslip angle.

4.3. Front Suspension Spring Stiffness. In Figure 9, the front suspension spring stiffness was randomly sampled with respect to the reference stiffness value and the $\pm 25 \%$ variation to investigate the sensitivity of the automobile stability and rollover responses in the frequency domain. Based on the frequency response amplitude in Figure 9(a), with a $\pm 25 \%$ variation in the front axle spring stiffness, the lateral acceleration bandwidth is not significantly influenced by the variation in the front suspension spring rate. However, as indicated in Figures 9(b)-9(d), the bandwidths of both the roll and sideslip angles are influenced by the $\pm 25 \%$ sampled spring stiffness. The peak amplitudes of the roll and front and rear sideslip angles increase by 13,21 , and $28 \%$, respectively. Note that increasing the spring stiffness decreases the roll and sideslip angles, which enhances the automobile rollover and stability and vice versa. It can also be inferred that changing the suspension stiffness, which can be achieved by variations in the materials, manufacturing processes, and vehicle type, has nearly no apparent effect on the modal resonant frequencies of the automobile stability and rollover responses.

4.4. Rear Suspension Spring Stiffness. Bandwidth analyses of how sensitive the automobile stability and rollover attitudes are regarding the rear suspension stiffness parameter are highlighted in Figure 10. In general, the automobile stability in terms of the lateral vehicle acceleration presents a low correlation and bandwidth sensitivity with respect to the $\pm 25 \%$ randomly sampled stiffness $\left(K_{\mathrm{sr}}\right)$. Otherwise, the bandwidth analyses in Figures 10(b)-5(d) illustrate the precise bandwidth sensitivity of the roll and sideslip angles' magnitudes, demonstrating a slight shift in their modal resonant frequencies. In Figure 10(b), the frequency response magnitude of the roll angle presents a nearly $9 \%$ drop in the peak magnitude value when the rear suspension stiffness $\left(K_{\mathrm{sr}}\right)$ increases by $25 \%$ with respect to the reference stiffness. Conversely, when the reference spring stiffness $\left(K_{\mathrm{sr}}\right)$ is reduced by $25 \%$, the roll angle magnitude increases by nearly $15 \%$, deteriorating the automobile rollover performance. Unlike the roll angle correlation with respect to the rear spring stiffness coefficient, both the front and rear sideslip angles present an inverse correlation with respect to the spring stiffness variation. The sideslip angle increases when the stiffness value increases and vice versa, and the automobile stability is inversely proportional to the suspension stiffness coefficient. Figures 8(c) and 8(d) show that the frequency response magnitudes of the front and rear sideslip angles increase by approximately 15 and 20\%, respectively, when the stiffness value $\left(K_{\mathrm{sr}}\right)$ increases by $25 \%$ with respect to the reference. Conversely, when the reference spring stiffness $\left(K_{\mathrm{sr}}\right)$ is reduced by $25 \%$, the front and rear sideslip angles' peak magnitudes decrease by nearly 13 and $17 \%$, respectively.

4.5. Front Tire Stiffness. Figure 11 reports a complete bandwidth sensitivity analysis of the sensitivities of the lateral acceleration, roll angle, and front and rear sideslip angle with respect to the front tire stiffness variation. In these simulation analyses, the front tire stiffness $\left(K_{\mathrm{tf}}\right)$ was randomly sampled with respect to the reference $K_{\mathrm{tf}}$ value and the $\pm 25 \%$ variation to investigate the bandwidth sensitivity response of the automobile rollover stability in the frequency domain. In general, all the automobile stability and rollover responses present higher bandwidth sensitivities in terms of the amplitude and modal resonant frequency bandwidths correlated to the tire stiffness variation, highlighting the significant influence of the tire stiffness on automobile stability and rollover.

The lateral acceleration bandwidth sensitivity analysis in Figure 11(a) indicates that the lateral acceleration is highly 


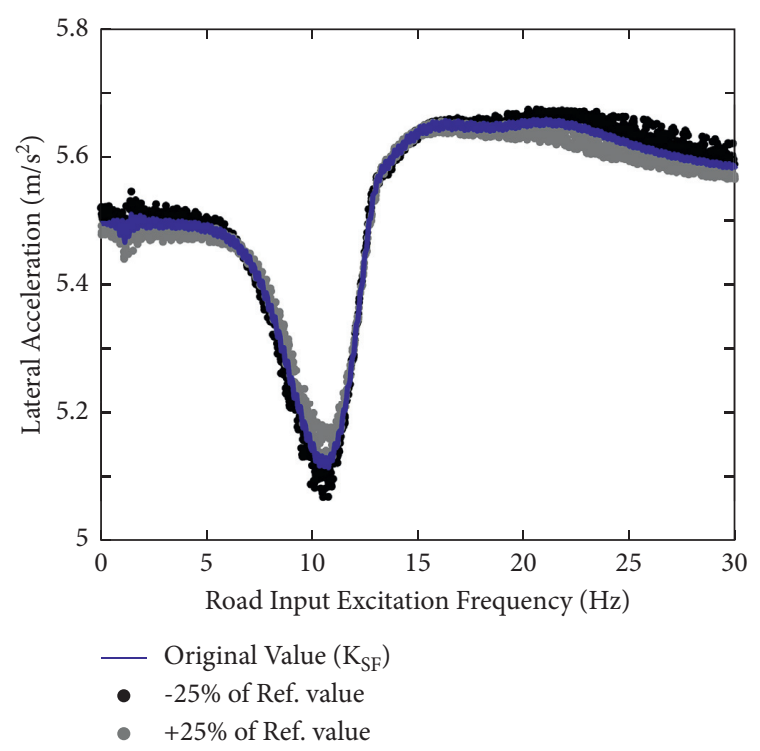

(a)

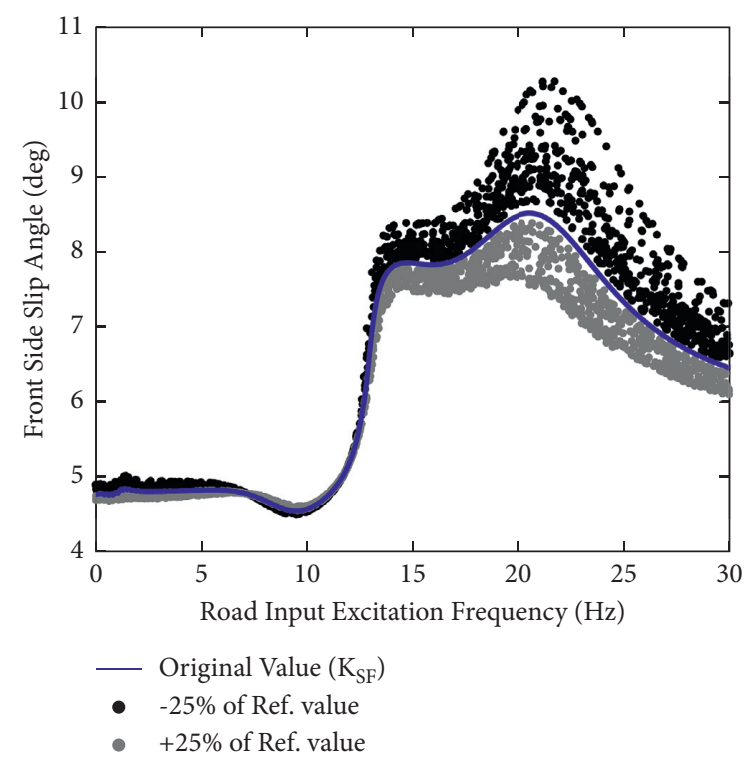

(c)

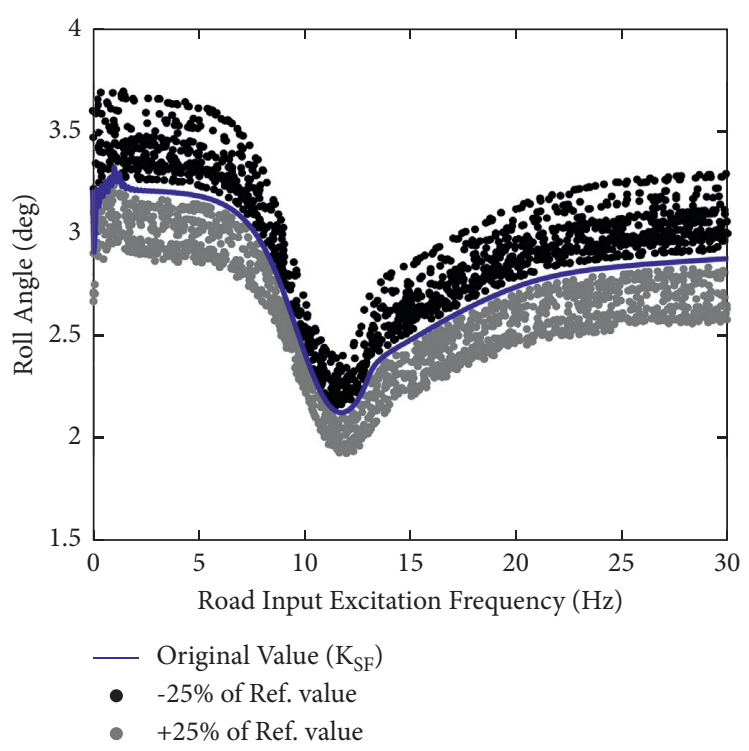

(b)

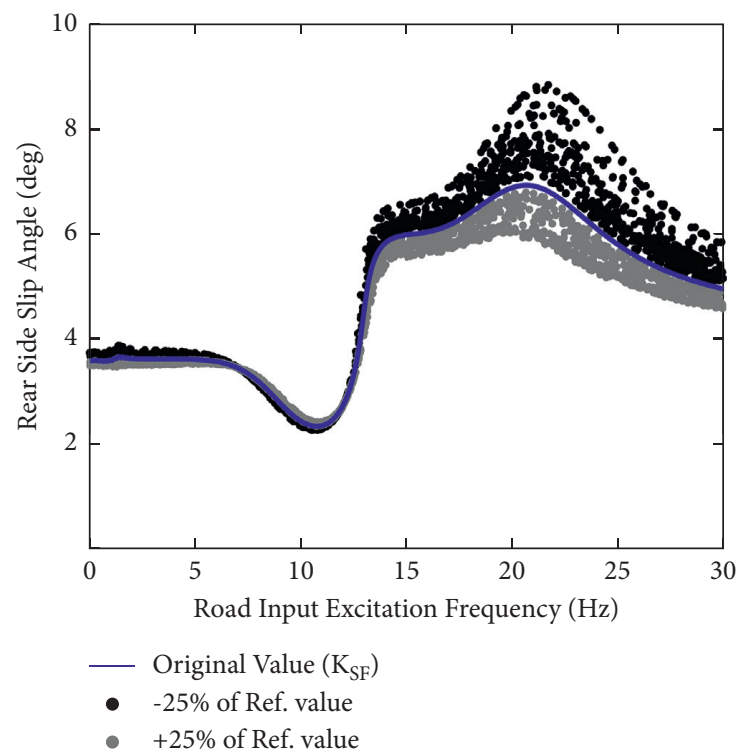

(d)

FIGURE 9: Sensitivity simulation results for automobile handling and stability responses with respect to the front spring stiffness coefficient parameter. (a) Lateral acceleration response; (b) roll angle response; (c) front sideslip angle response; (d) rear sideslip angle.

correlated to the vehicle tire stiffness. The lateral vehicular stability is inversely proportional to the vertical tire stiffness coefficient $\left(K_{\mathrm{tf}}\right)$. The lateral acceleration magnitude decreases from $5.11 \mathrm{~m} / \mathrm{s}^{2}$ to $4.55 \mathrm{~m} / \mathrm{s}^{2}$, a nearly $11 \%$ drop in the peak magnitude value, when the front tire stiffness $\left(K_{\mathrm{tf}}\right)$ is increased by $25 \%$. The model resonant frequency corresponding to the lateral vehicle acceleration's peak magnitude is straightforwardly shifted from 10.52 to $12.77 \mathrm{~Hz}$. Conversely, when the reference tire stiffness $\left(K_{\mathrm{tf}}\right)$ is reduced by $25 \%$, the lateral acceleration magnitude increases by nearly $3 \%$, deteriorating the automobile lateral stability. Similar to the lateral acceleration sensitivity performance, the roll angle response is strongly correlated with the tire stiffness variation, including an apparent shift in its modal resonant frequency. The automobile roll angle appears to have an inversely proportional correlation with the tire stiffness, as seen in Figure 11(b). When $K_{\mathrm{tf}}$ is decreased by $25 \%$, the roll angle's peak amplitude increases by nearly $26 \%$, accompanied by a $1.32 \mathrm{~Hz}$ rearward shift in its modal resonant frequency. Conversely, when the reference value of $K_{\mathrm{tf}}$ is increased by $25 \%$, the magnitude of the roll angle response decreases by approximately $33 \%$, accompanied by a $0.93 \mathrm{~Hz}$ forward shift in its resonant frequency. Identical to the correlations of both the lateral acceleration and the roll angle with respect to the $\pm 25 \%$ randomly sampled tire stiffness, the sideslip angles and the tire stiffness coefficient are inversely proportional, as confirmed in Figures 11(c) and 11(d). However, when $K_{\mathrm{tf}}$ is decreased by $25 \%$, the first modal peak 


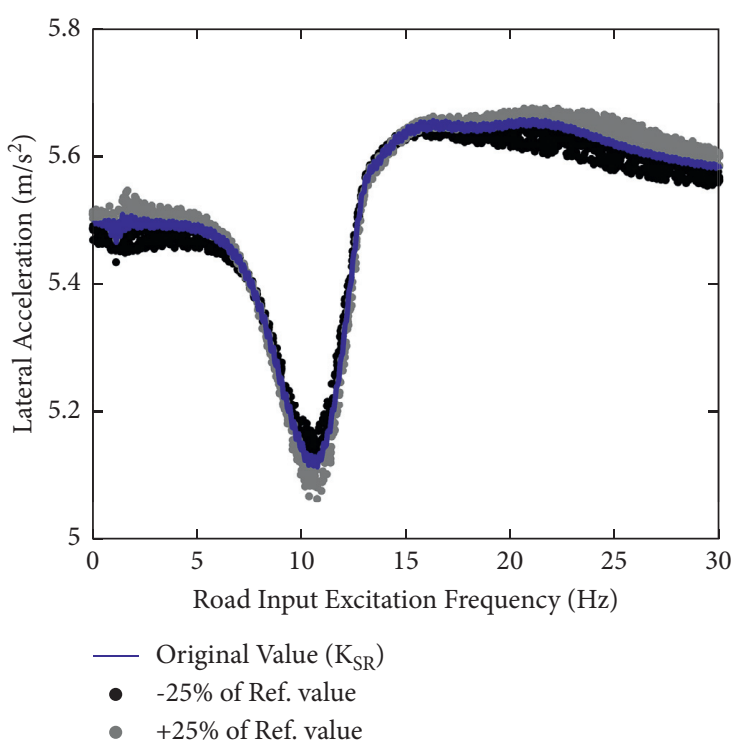

(a)

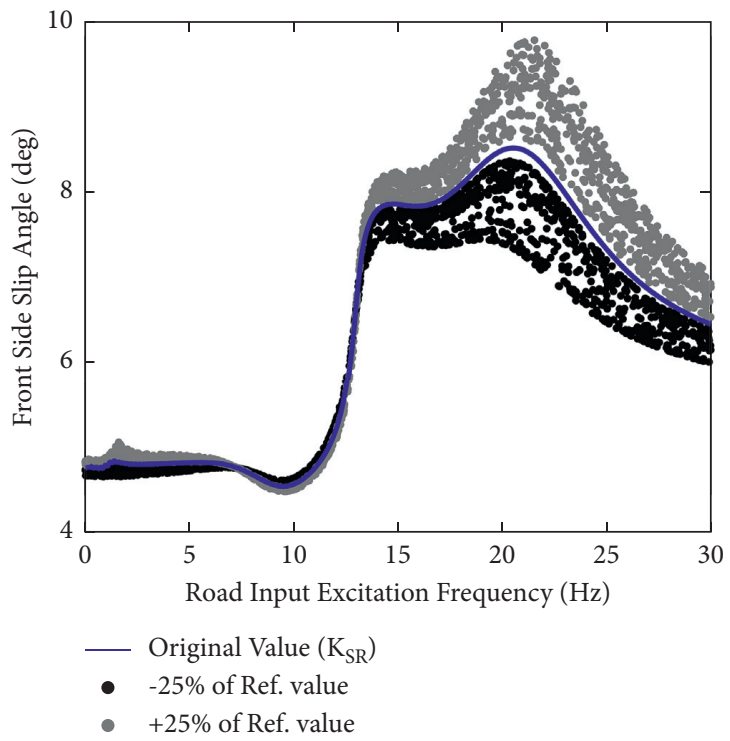

(c)

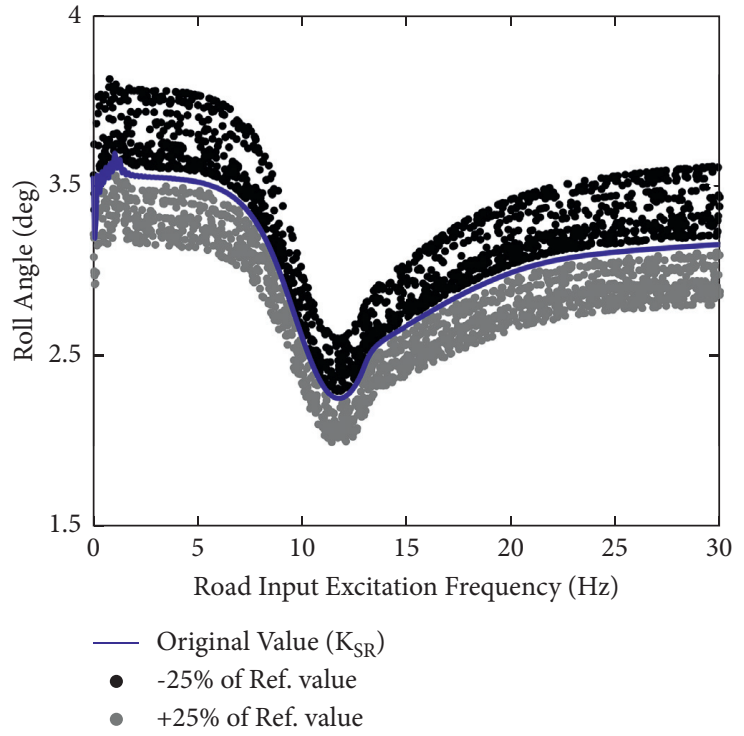

(b)

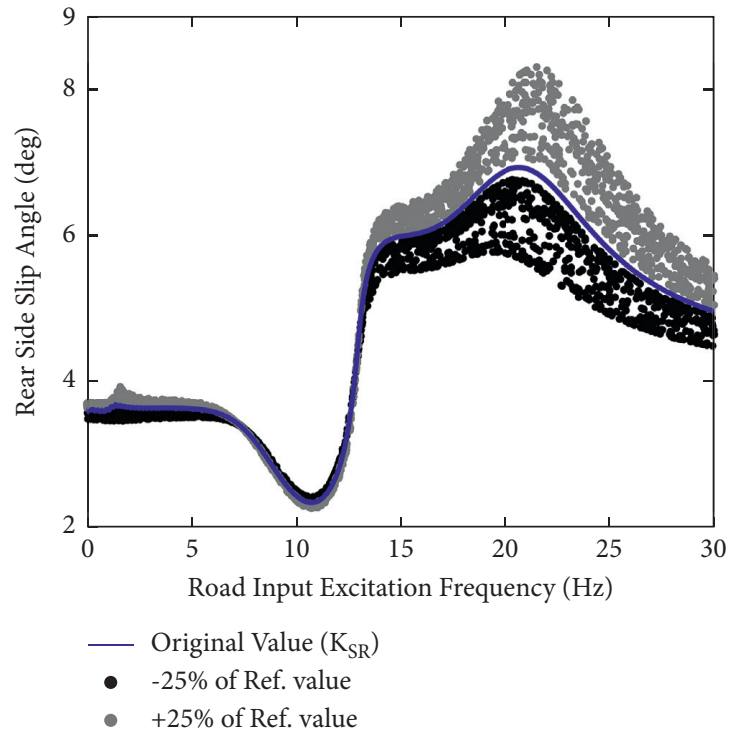

(d)

FIGURE 10: Sensitivity simulation results for automobile handling and stability responses with respect to the rear spring stiffness coefficient parameter. (a) Lateral acceleration response; (b) roll angle response; (c) front sideslip angle response; (d) rear sideslip angle.

magnitudes of the front and rear sideslip angles increase by nearly 31 and 53\%, respectively. Meanwhile, the secondmodal-peak magnitudes of the front and rear sideslip angles increase by nearly 256 and $376 \%$, respectively, deteriorating the automobile stability.

This is related to the tire contact patch, which decreases with higher tire stiffnesses, leading to a decrease in the tread's cornering stiffness. If the front cornering stiffness of the vehicle is smaller, the vehicle will experience understeering behavior, making the vehicle exhibit a broader understeer tendency [47]. We can infer that better vehicular directional stability is provided by a larger restoring moment coefficient and a smaller steering stiffness, and vice versa.
4.6. Rear Tire Stiffness. The sensitivities of the automobile stability and rollover attitudes to the rear tire stiffness $\left(K_{\mathrm{tr}}\right)$ are presented in Figure 12 . Here, $K_{\mathrm{tr}}$ was randomly sampled, similar to the parameters mentioned above, based on a $\pm 25 \%$ variation rate and the reference tire stiffness $\left(K_{\mathrm{tr}}\right)$ with respect to a randomly sampled excitation frequency. According to the frequency/amplitude response bandwidth plots provided in Figure 12, the automobile stability and rollover responses, except the roll angle response, indicate a clear sensitivity to the amplitude and the modal resonant frequency bandwidth correlated with the variation in the rear tire stiffness. In Figure 12(a), in the model resonant frequency range, the magnitude of the lateral acceleration decreases from 5.128 to $5.04 \mathrm{~m} / \mathrm{s}^{2}$, a nearly $2 \%$ drop 


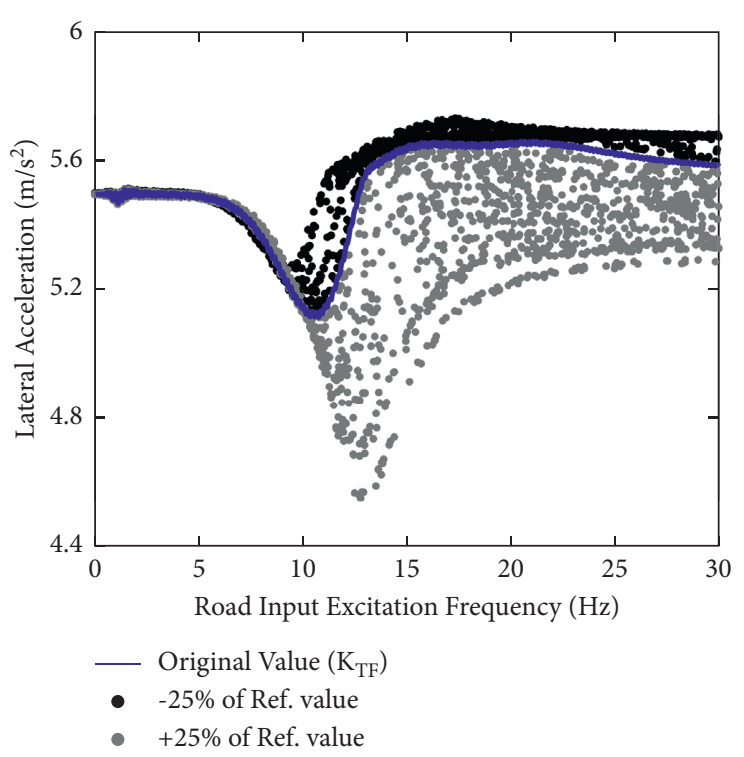

(a)

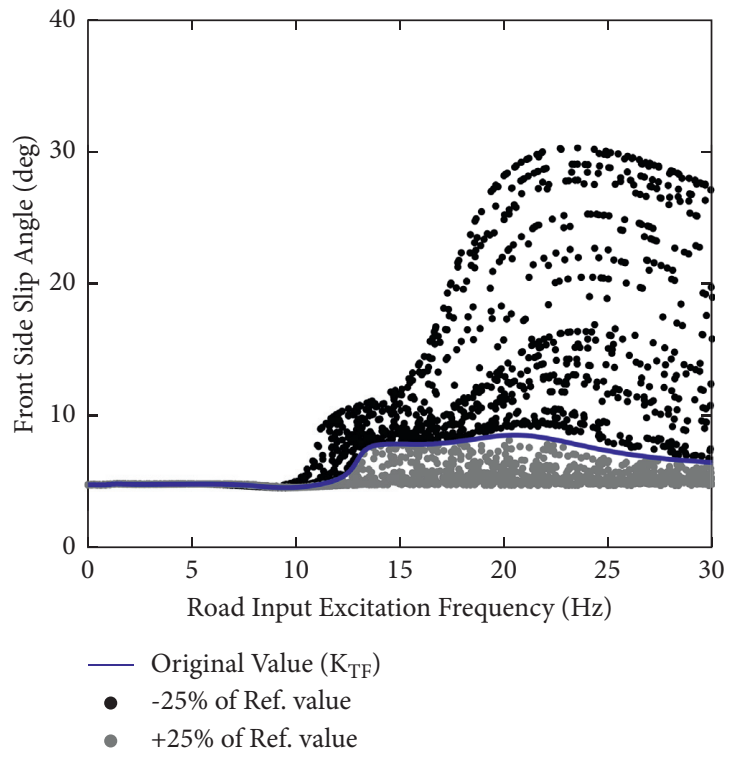

(c)

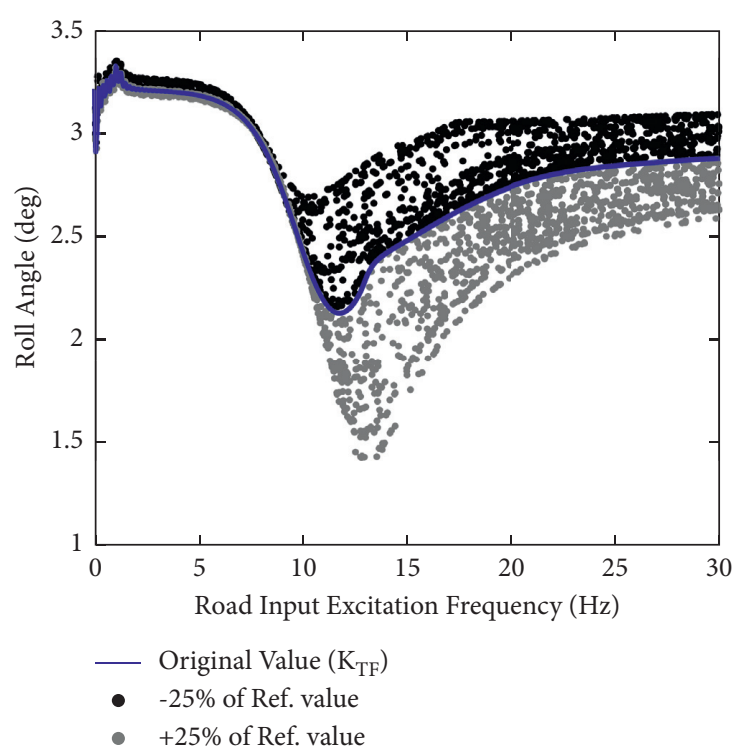

(b)

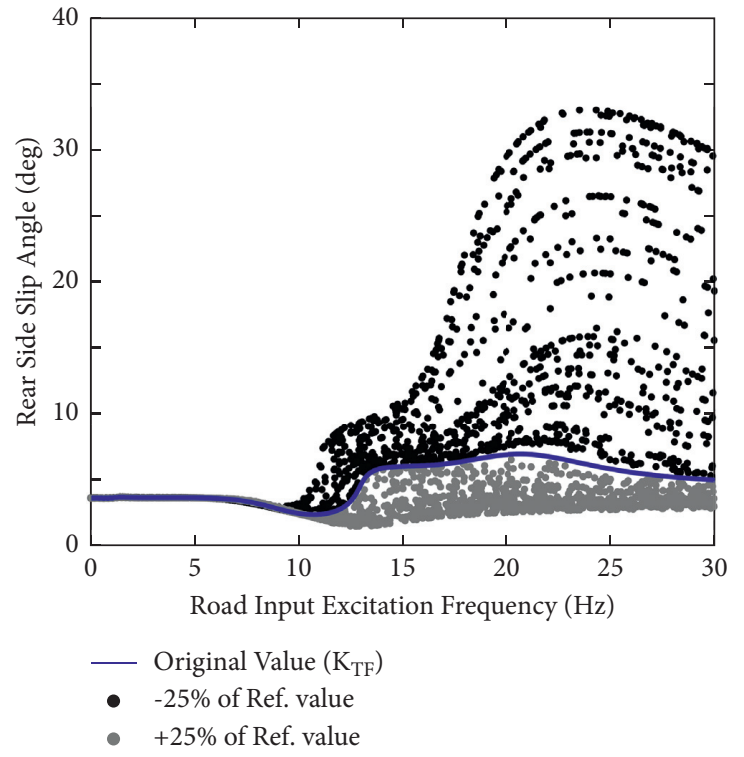

(d)

FIGURE 11: Sensitivity simulation results for automobile handling and stability responses with respect to the front tire stiffness coefficient parameter. (a) Lateral acceleration response; (b) roll angle response; (c) front sideslip angle response; (d) rear sideslip angle.

corresponding to a $25 \%$ increase in $K_{\text {tr }}$, and from 5.128 to $4.84 \mathrm{~m} / \mathrm{s}^{2}$, a nearly $4 \%$ drop corresponding to a $25 \%$ decrease in $K_{\mathrm{tr}}$. Unlike the roll angle's sensitivity to the front tire stiffness variation, the roll angle barely responds to the variation in the rear tire stiffness during the sensitivity simulations (see Figure 12(b)).

As illustrated in Figures 12(c) and 12(d), when $K_{\mathrm{tr}}$ is increased by $25 \%$, the front and rear sideslip angles' modal peak magnitudes increase by nearly 24 and 36\%, respectively. However, when $K_{\text {tr }}$ is decreased by $25 \%$, the front and rear sideslip angles' peak magnitudes decreased by nearly 51 and $72 \%$, respectively, deteriorating automobile stability.

In conclusion, it is inferred that changing the tire stiffness, which could be done by the variations of materials, construction, and manufacturing processes, and vehicle type has a clear and significant effect on the automobile stability and rollover responses in terms of the bandwidth of both the magnitudes and modal resonant frequencies.

4.7. Longitudinal C.G. Position. Figures 13 and 14 illustrate bandwidth sensitivity analyses of the automobile stability and rollover attitudes versus longitudinal and vertical C.G. positions. The magnitude bandwidth of the lateral acceleration, roll angle, and sideslip angles present higher sensitivity correlations than the longitudinal C.G. position and the C.G. height. Figure 13 shows the effect of shifting C.G. either forward or rearward by $\pm 25 \%$ with 


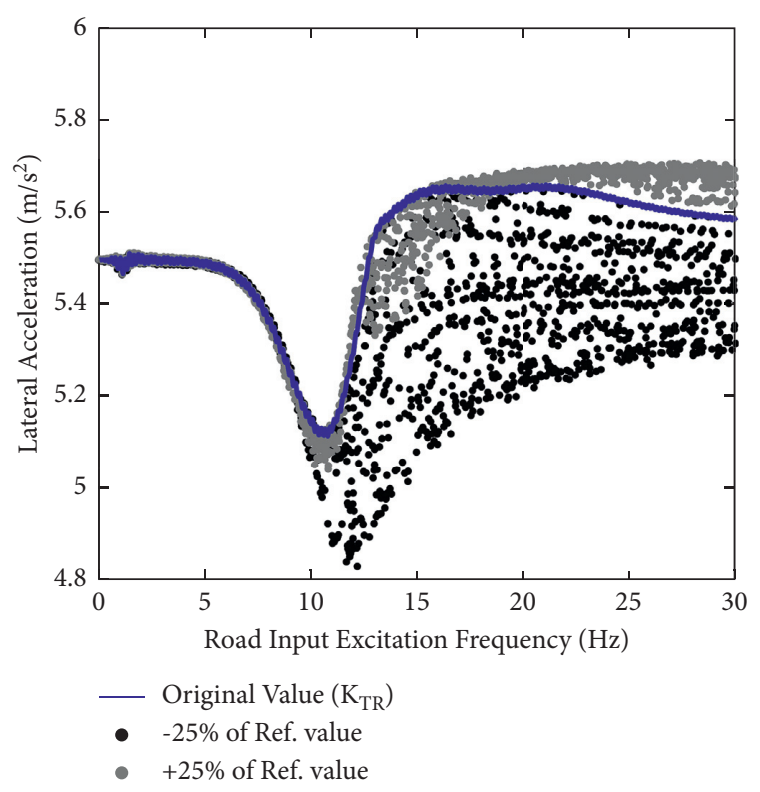

(a)

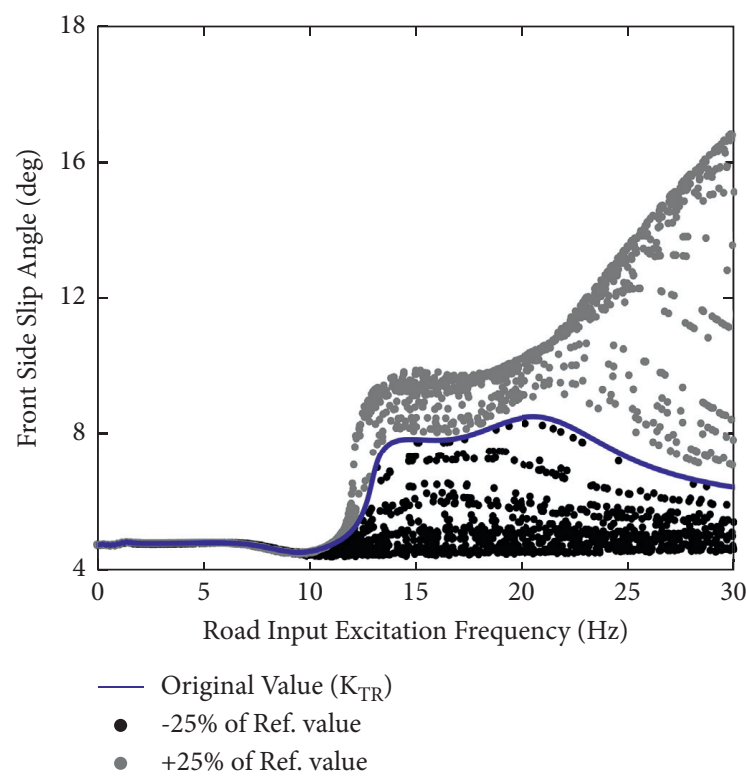

(c)

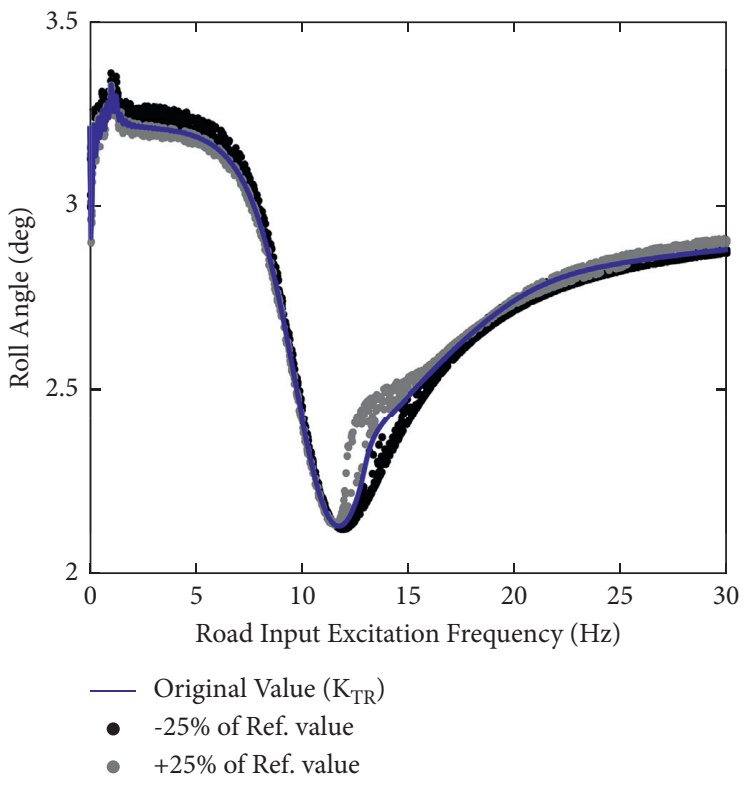

(b)

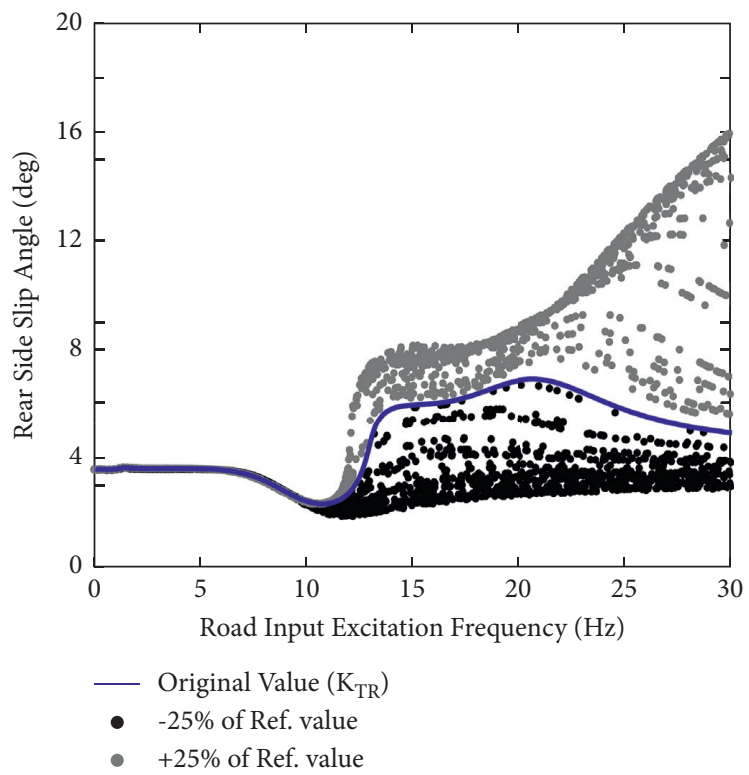

(d)

FIGURE 12: Sensitivity simulation results for automobile handling and stability responses with respect to the rear tire stiffness coefficient parameter. (a) Lateral acceleration response; (b) roll angle response; (c) front sideslip angle response; (d) rear sideslip angle.

respect to its reference position for randomly sampled excitation frequencies. In simulations, a $+25 \%$ variation implies the C.G. is shifted rearward to the rear axle, while a $-25 \%$ means that the C.G. is shifted toward the front axle. According to the frequency/amplitude response bandwidth plots provided in Figure 13, the automobile stability and rollover responses exhibited an apparent sensitivity to the longitudinal C.G. distance variation. Moving C.G. forward ( $-25 \%$ with respect to the reference C.G. position) resulted in superior stability and rollover dynamics because all automobile stability and rollover responses decrease with the forward shifting of the C.G. position of a vehicle. Conversely, when C.G. is rearward shifted $(+25 \%$ with respect to the reference C.G. position), the lateral acceleration, roll angle, and front and rear sideslip angles increase, deteriorating the automobile stability and rollover dynamics. Figures 13(c) and 13(d) correspond to a $25 \%$ rearward shift in C.G., the peak magnitudes of the front and rear sideslip angles increase by nearly 255 and $478 \%$, respectively. Conversely, with a $25 \%$ forward shift in C.G., the front and rear sideslip angles' peak magnitudes decrease by nearly 24 and 35\%, respectively, improving the automobile stability. The investigated automobile stability and rollover responses 


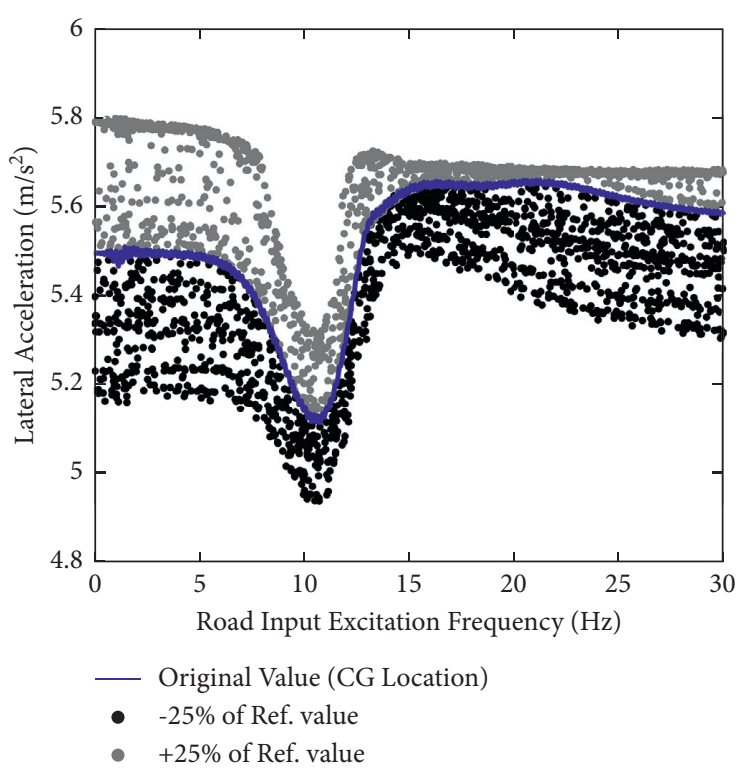

(a)

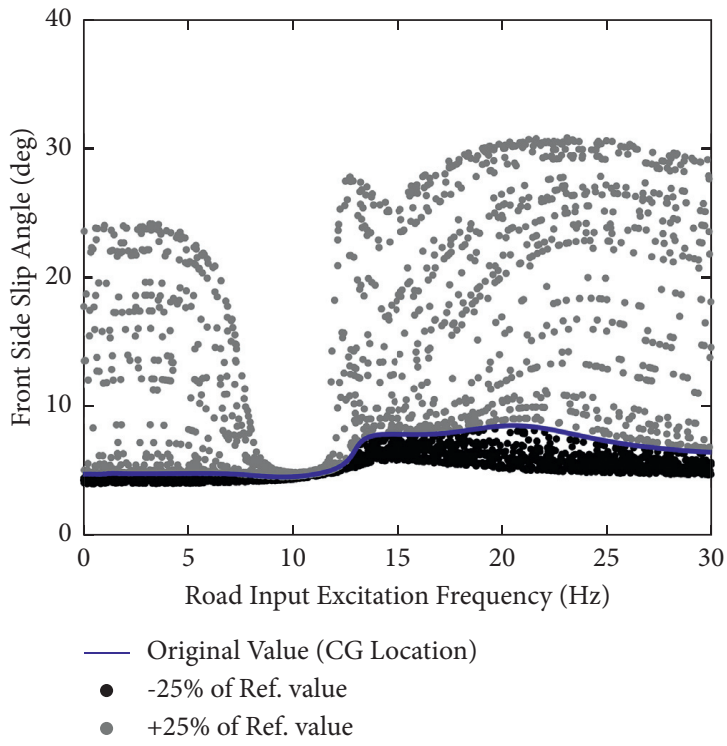

(c)

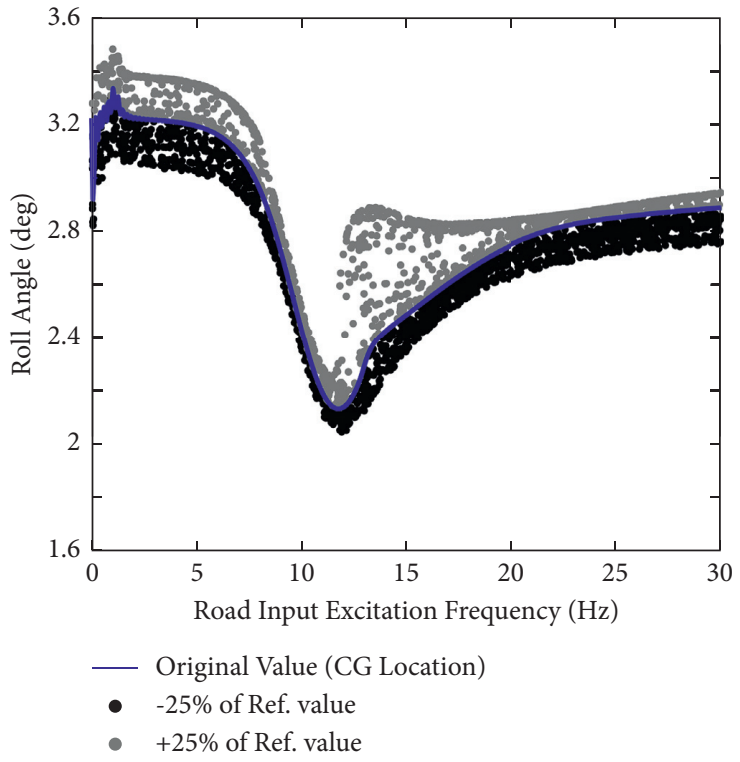

(b)

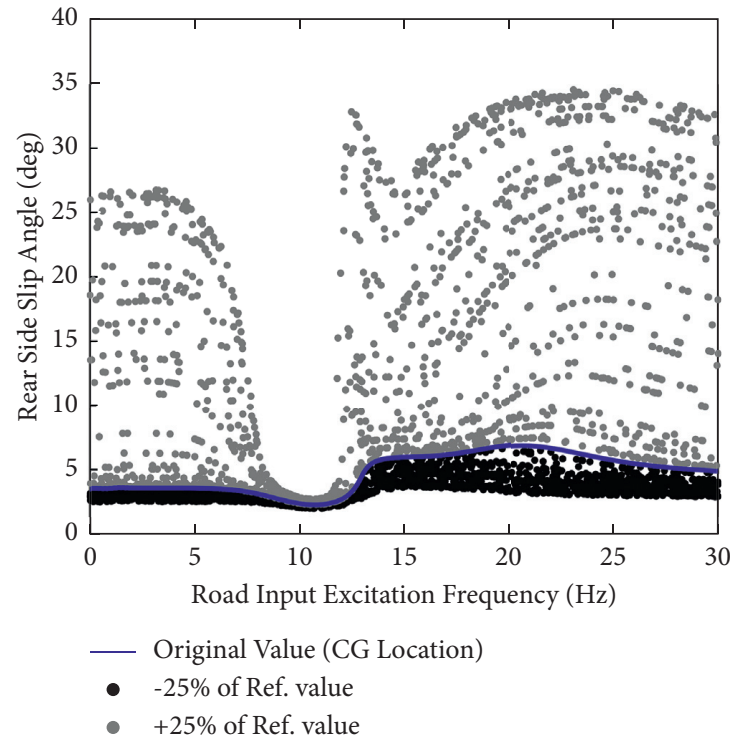

(d)

FIGURE 13: Sensitivity simulation results for automobile handling and stability responses with respect to the longitudinal C.G. position parameter. (a) Lateral acceleration response; (b) roll angle response; (c) front sideslip angle response; (d) rear sideslip angle.

illustrate no apparent effects of the frequency bandwidth of the model resonant frequencies on the lateral acceleration, roll angle, and front and rear sideslip angles.

4.8. C.G. Height. The sensitivities of the automobile stability and rollover dynamics versus the C.G. height of the vehicle are shown in Figures 14. Figure 14 demonstrates the inconsistent effects of the C.G. height, where the lateral acceleration trend decreases with respect to the increasing C.G. height of the vehicle, in opposition to the roll angle trends and front and rear sideslip angles. According to the magnitude bandwidth of the vehicle sideslip angles in Figures 14(c) and 14(d), the closer C.G. is to the ground, the better the rollover stability and vice versa. When the vehicular C.G. height is increased by $+25 \%$ related to the reference vertical C.G. position, the peak magnitudes of the front and rear vehicle sideslip angles increase by approximately 30 and $40 \%$, respectively. Conversely, when the vehicular C.G. height is decreased by $-25 \%$, the peak magnitudes of the front and rear sideslip angles decrease by nearly 15 and $18 \%$, respectively, improving the automobile stability. Note that there is no significant or apparent change in the model resonant frequencies of the sideslip angles' peak magnitudes. 


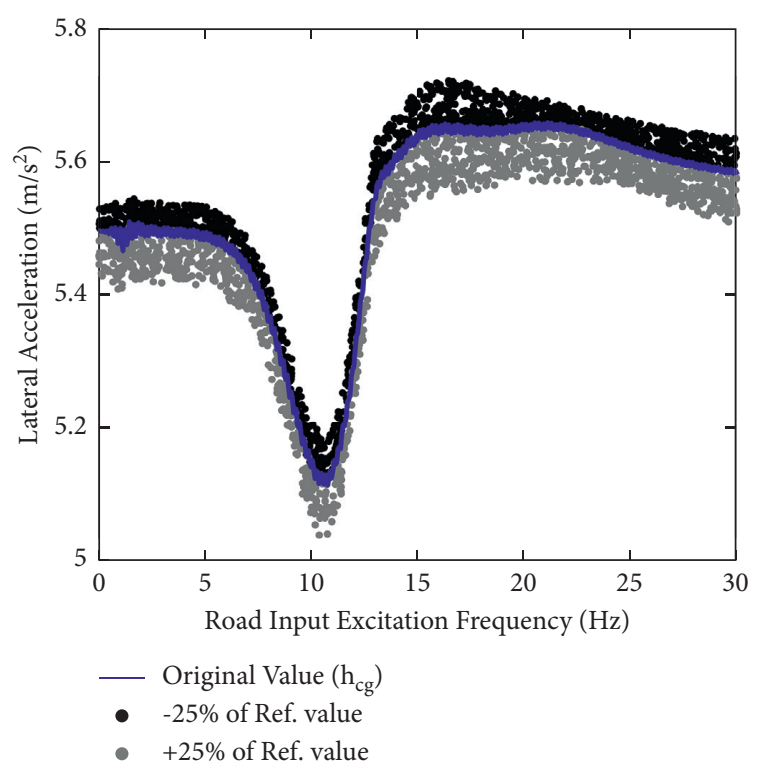

(a)

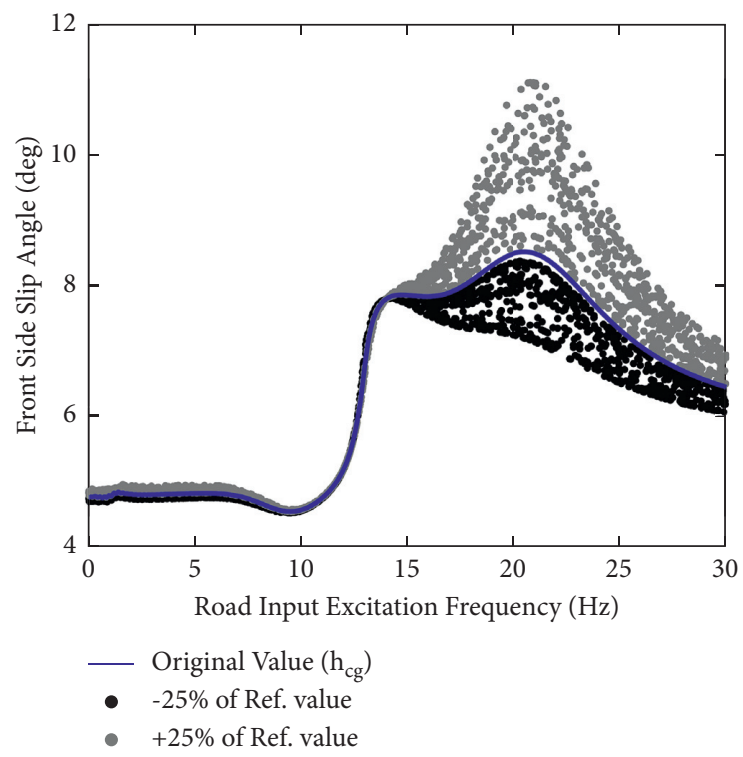

(c)

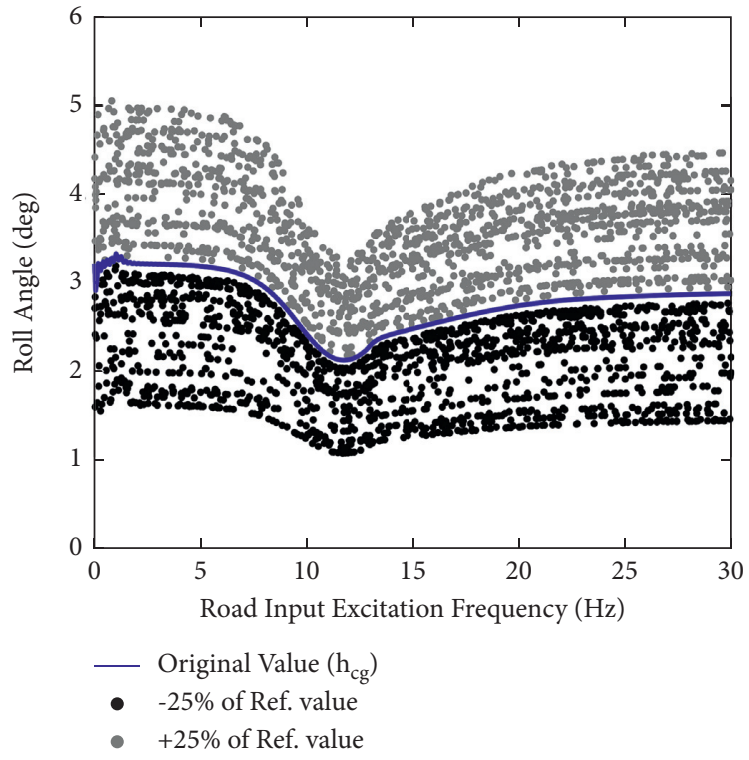

(b)

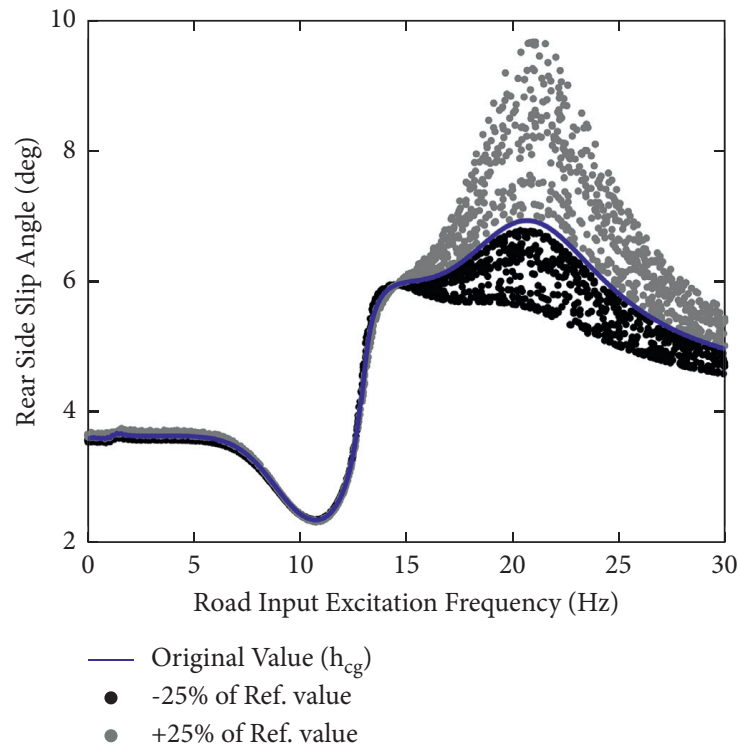

(d)

FIGURE 14: Sensitivity simulation results for the automobile handling and stability responses with regard to the C.G. height parameter. (a) Lateral acceleration response; (b) roll angle response; (c) front sideslip angle response; (d) rear sideslip angle.

4.9. Wheelbase Length. Figure 15 illustrates the sensitivities of the automobile stability and rollover dynamics to the wheelbase parameter. According to the sensitivity results provided in Figure 15, the vehicle wheelbase length exerts a considerable influence on the lateral acceleration and sideslip angles. Meanwhile, the wheelbase length variation only slightly influences the vehicular rollover response, as shown in Figure 15(b). In Figure 15(a), the lateral acceleration trend decreases with respect to increasing wheelbase length, particularly at low frequencies. However, the front and rear sideslip angles decrease significantly at high frequencies with higher wheelbase lengths, as shown in Figures 15(c) and 15(d). When the vehicle wheelbase length is increased by $25 \%$ with respect to its reference value, the peak magnitudes of the front and rear vehicle sideslip angles decrease by approximately 25 and 17\%, respectively.

Conversely, when the vehicle wheelbase length is decreased by $25 \%$, the peak magnitudes of the front and rear sideslip angles increase by nearly 31 and 29\%, respectively, improving the automobile stability. These results confirm the association between the wheelbase length and the overall vehicular lateral and roll stabilities. Therefore, vehicles with longer wheelbase lengths provide better overall stability than those with comparatively short wheelbase lengths.

Figure 16 summarizes the bandwidth sensitivity results for the automobile handling and stability criteria with $\pm 25 \%$ 


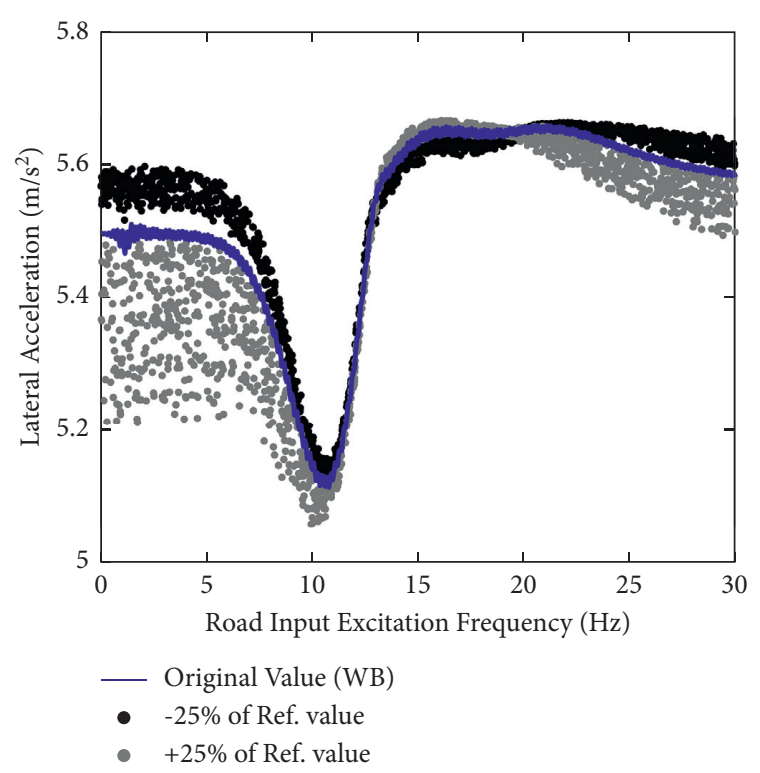

(a)

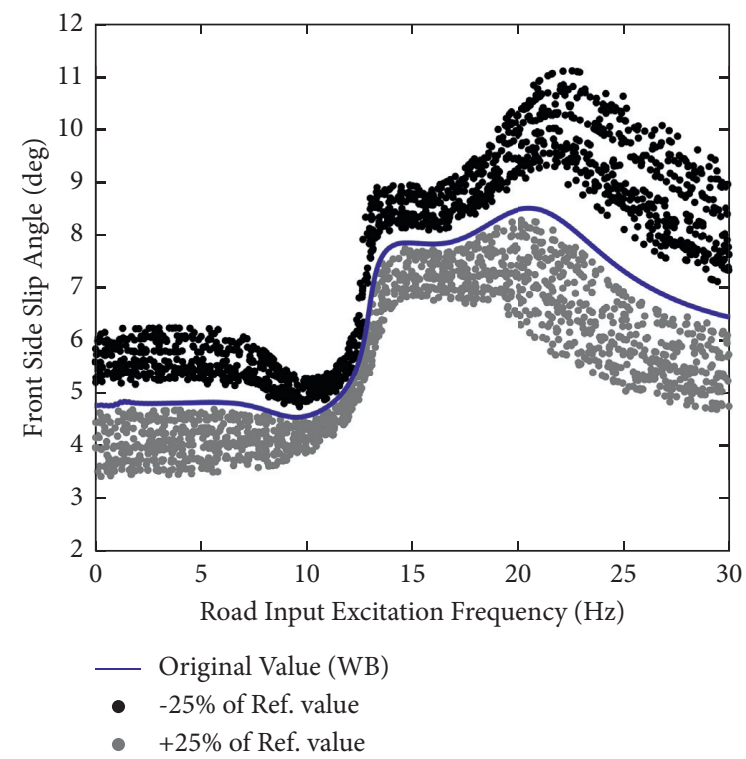

(c)

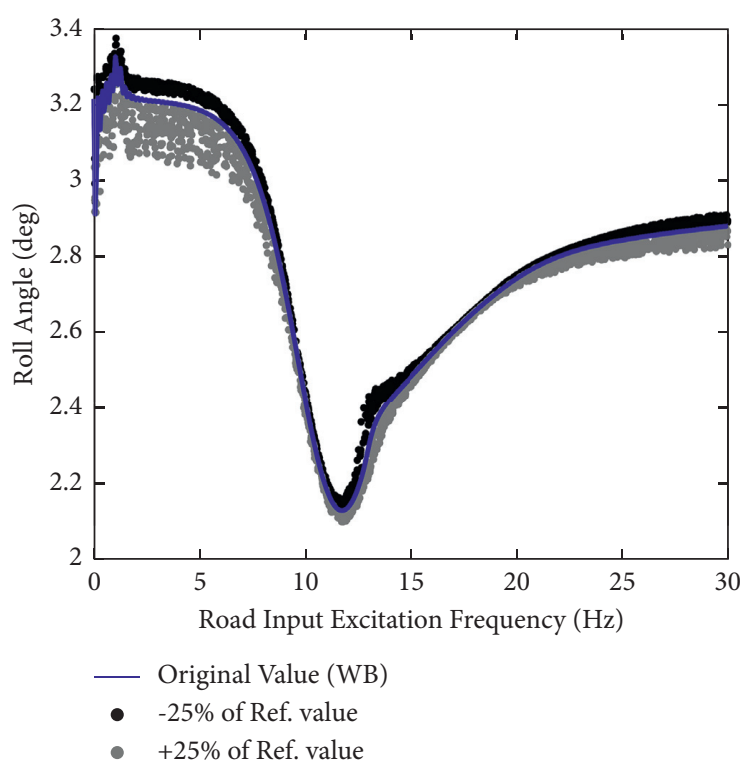

(b)

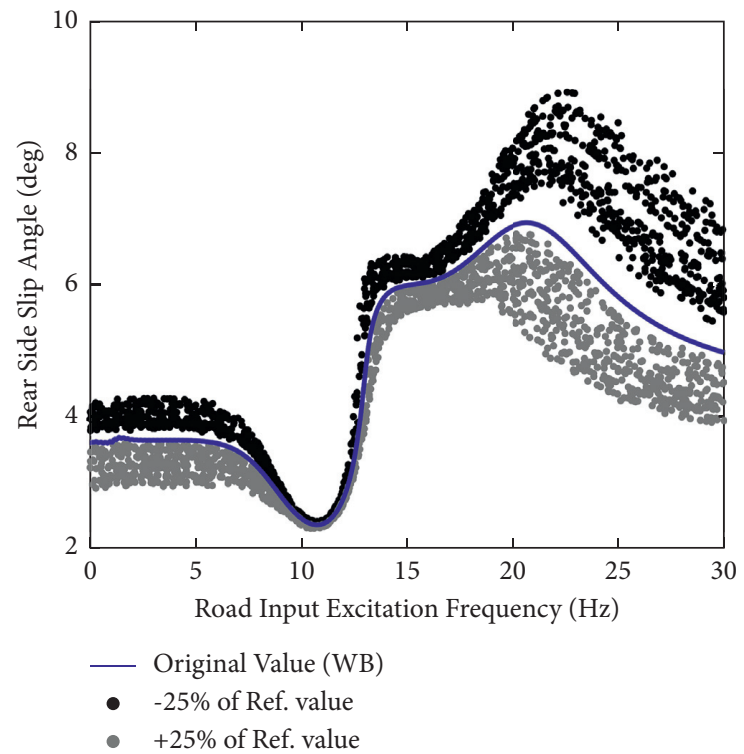

(d)

FiguRE 15: Sensitivity simulation results for automobile handling and stability responses with respect to the wheelbase length parameter. (a) Lateral acceleration response; (b) roll angle response; (c) front sideslip angle response; (d) rear sideslip angle response.

variations in the influential vehicle parameters with respect to the reference parameters. Figures $16(\mathrm{a})$ and $16(\mathrm{~b})$ indicate that, for a $-25 \%$ variation in the tire stiffness, the magnitudes of the modal amplitudes corresponding to the rear and front sideslip angles significantly increase by $400 \%$ and $280 \%$, respectively. Conversely, the roll angle, yaw rate, and lateral acceleration present lower sensitivity changes in their peak amplitudes with a tire stiffness variation of $-25 \%$ with respect to the nominal tire stiffness value. In Figures 16(c) and 16(d), when C.G. is rearward shifted by $25 \%$ with respect to the default position, the modal resonant frequencies of the front and rear sideslip angles correlate strongly with this shift compared to the responses of the remaining criteria.
The wheel track presents a minor effect on the frequency bandwidths for the lateral acceleration, roll angle, yaw rate, and sideslip angles.

At the first modal resonant frequency, with a $-25 \%$ variation in the nominal vehicle parameters, the front and rear tire stiffnesses and the rear damping coefficient significantly affect the peak resonant frequency shift. It is apparent from Figure 17(a) that the modal resonant frequencies of the front and rear sideslip angles move forward by 22 and $21 \%$, respectively, with the variation in the front tire stiffness. The roll angle and yaw rate peaks shift by approximately $12 \%$ with the decreased rear damping coefficient. The results of the $+25 \%$ variations in the reference 


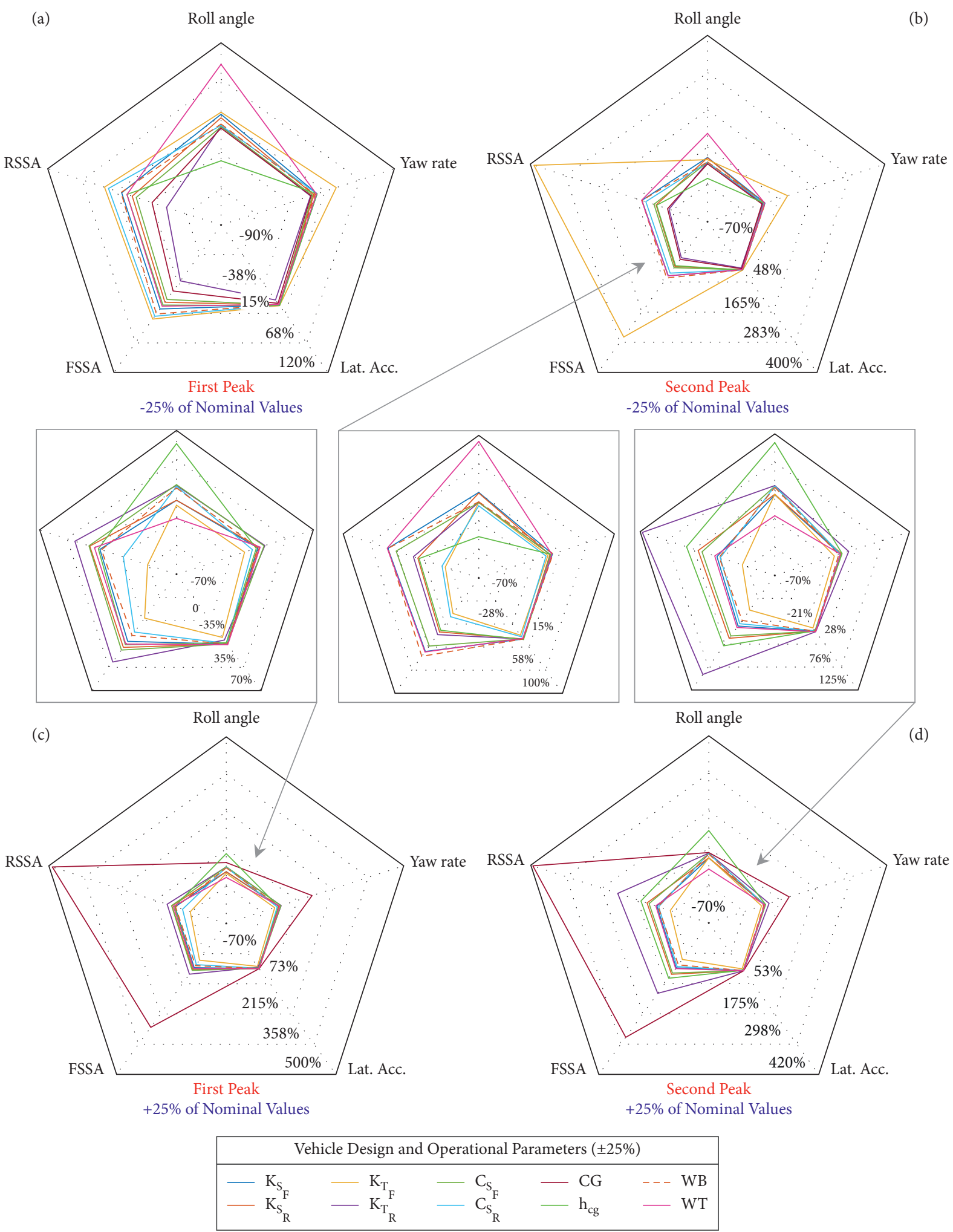

FIGURE 16: Summary of the sensitivity analyses of the handling and stability criteria versus the vehicle parameters: $(a, b)$ the percentage of change of both first- and second-modal-peak at $-25 \%$ of nominal vehicle parameters; (c, d) percentage of change of both first- and secondmodal-peak at $+25 \%$ of nominal vehicle parameters (FSSA: front sideslip angle, RSSA: rear sideslip angle, Lat. Acc.: lateral acceleration).

vehicle parameters indicate that the vehicle parameters have equal effects on the shift in the roll angle peak, ranging from 13 to $16 \%$. However, the front tire stiffness significantly influences the yaw rate resonant frequency by $25 \%$ compared to the other vehicle parameters. Furthermore, the lateral acceleration's modal resonant frequency is not 


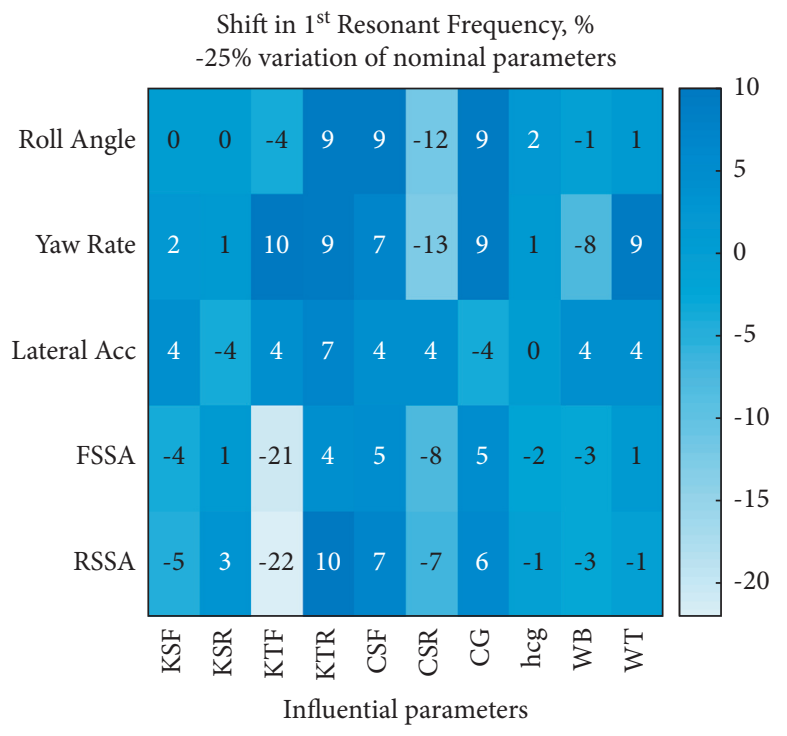

(a)

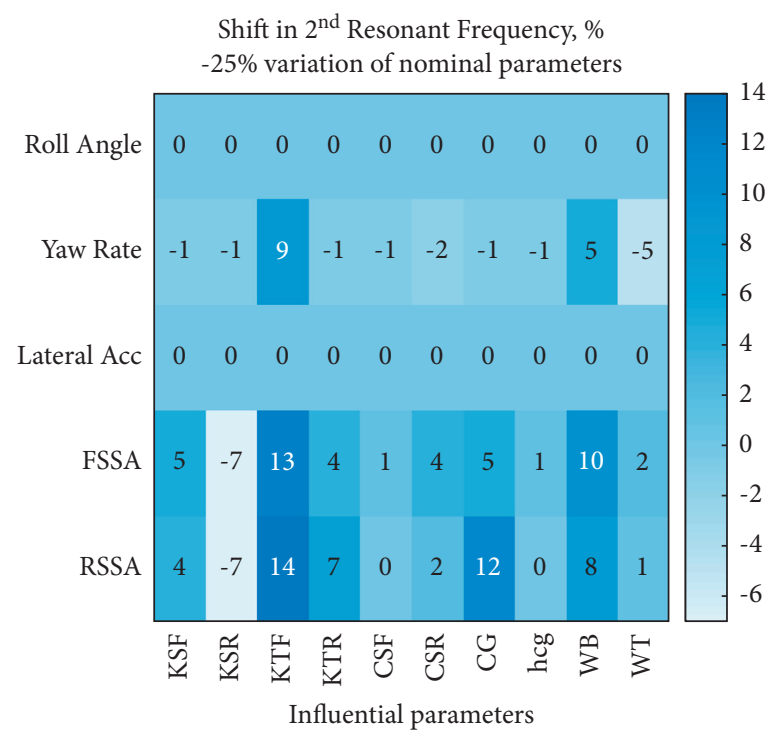

(c)

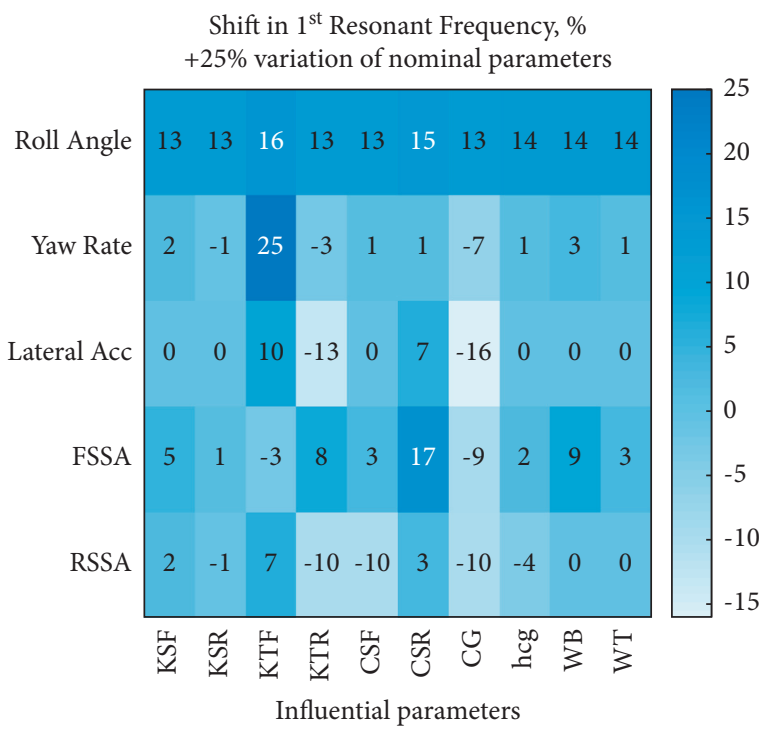

(b)

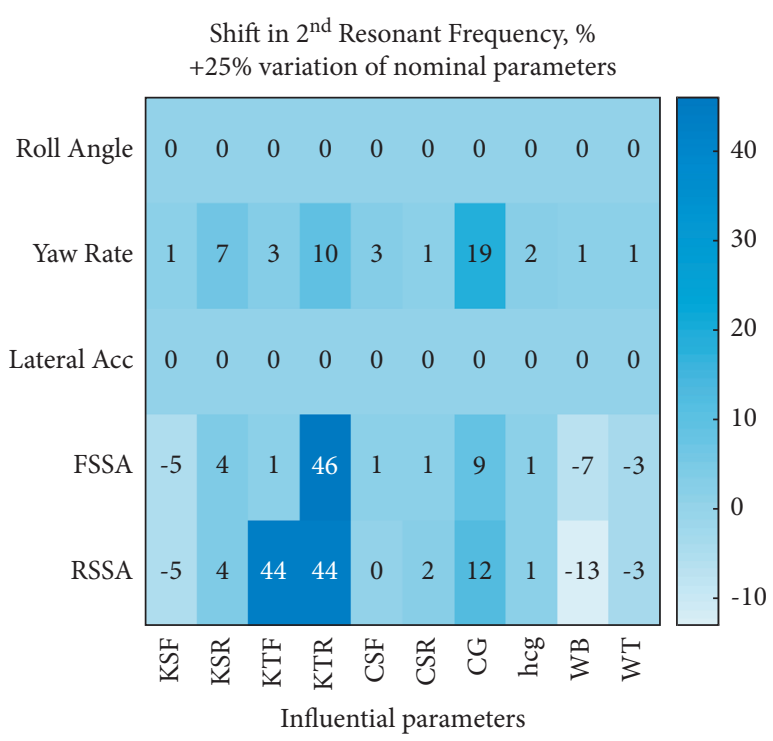

(d)

FIGURE 17: Sensitivity analyses of the resonant frequency peaks versus the vehicle parameters: $(a, b)$ percentage of change of the first modalresonant frequency at $\pm 25 \%$ of nominal vehicle parameters; $(c, d)$ percentage of change of the modal-resonant frequency at $\pm 25 \%$ of nominal vehicle parameters (FSSA: front sideslip angle, RSSA: rear sideslip angle, Lat. Acc.: lateral acceleration).

changed by variations in the suspension stiffness, C.G. height, wheelbase length, and wheel track.

A frequency bandwidth analysis of the lateral and roll stability responses versus the vehicle parameter variations is shown in Figures 17(c) and 17(d). At the second modal resonant frequency, the tire stiffness, longitudinal C.G. position, and wheelbase length are the most influential parameters for the frequency bandwidths of the front and rear sideslip angles. The front tire stiffness plays an essential role in the front and rear sideslip angles and the yaw rate of the abovementioned parameters.
Conversely, decreasing the vehicle parameters by $25 \%$ with respect to their nominal values does not affect the second modal resonant frequency of the roll angle and lateral acceleration, as shown in Figure 17(c). Figure 17(d) indicates that increasing the rear tire stiffness has the most significant effect, overwhelming all other parameters for the front and rear tire sideslip angles. Similarly, the front tire stiffness demonstrates similar behavior to the rear tire sideslip angle. The yaw rate, C.G. longitudinal position, rear tire stiffness, and rear suspension stiffness have considerable frequency 
bandwidth sensitivity. At the same time, the influence of higher vehicle parameter values on the roll angle and lateral acceleration are indisputable.

\section{Conclusions}

This study extensively conducted a parametrical bandwidth sensitivity analysis of the vehicular lateral/roll stability, including vehicle geometry, tire properties, and suspension parameters. In this approach, a 7-DOF fullvehicle model combined with a 2 -DOF planar two-track model with a nonlinear Pacejka tire model was developed. This study mainly provides an investigation of both the frequency and amplitude bandwidth sensitivity of the CG location, wheelbase length, wheel track width, suspension characteristics, and front/rear tire spring stiffness, and their effect on lateral stability and rollover propensity based on the Monte Carlo simulations. With regard to the uniformly randomized excitation frequency ranging between 0 and $30 \mathrm{~Hz}$, the selected parameters were typically randomized according to the Gaussian probability distribution based on their nominal values and a $25 \%$ standard deviation. During the sensitivity simulations, the peak and RMS values of the lateral acceleration, roll angle, and front/rear sideslip angles are calculated considering the uniformly randomized frequency.

The simulation results confirm that road excitation, tire properties, vehicle geometry, and suspension parameters strongly influence the vehicular lateral and roll stabilities, accounting for the lower and higher peaks and frequency bandwidths of these stabilities. Therefore, it appears that the roll angle response is strongly correlated with low-frequency road excitations. Conversely, the front/rear sideslip angles and the lateral acceleration are strongly influenced when the vehicle is subjected to highfrequency road excitations. The second major finding was that the tire properties and the longitudinal C.G. location significantly affect the vehicular lateral stability. Thus, the roll stability illustrates a high parametric sensitivity to the vertical C.G. position and the wheel track width.

These results can be illustrated as follows: when the front tire stiffness is decreased by $25 \%$ with respect to its standard value, the first peaks of the front and rear sideslip angles increase by 36 and 50\%, respectively, while the second peaks increase sharply by 275 and $380 \%$, respectively. Conversely, the increases in the first peaks of the front and rear sideslip angles with a $+25 \%$ variation in the rear tire stiffness are 29 and $30 \%$ from the nominal values, respectively, while the increases in the second peaks are 100 and $129 \%$ from the nominal values, respectively. A $25 \%$ rearward shift in the longitudinal C.G. position increases the first and second peak magnitudes of the front and rear sideslip angles by nearly 255 and $478 \%$, respectively. The above discussion demonstrates that higher values of the front tire stiffness and lower rear tire stiffness values provide better vehicular lateral stability. When the longitudinal C.G. position is located closer to the front axle, this improves vehicle stability. Choosing the correct tire properties, vehicle geometry, and suspension characteristics are essential in increasing the vehicular lateral stability and the rollover threshold. The highlighted variations in the abovementioned parameters will allow higher vehicular stability when a vehicle is excited by random road surfaces. Finally, the findings of this frequency-based bandwidth analysis are of great interest to future control approaches to enhance the lateral vehicle stability and rollover threshold.

\section{Data Availability}

The data that support the findings of this study are available from the corresponding author upon reasonable request.

\section{Conflicts of Interest}

The authors declare no conflicts of interest with respect to the research, authorship, and/or publication of this article.

\section{References}

[1] R. Allen, D. Klyde, T. Rosenthal, and D. Smith, "Estimation of passenger vehicle inertial properties and their effect on stability and handling," SAE Technical Paper 2003-01-0966, pp. 1032-1050, 2003.

[2] R. Whitehead, W. Travis, D. Bevly, and G. Flowers, "A study of the effect of various vehicle properties on rollover propensity," SAE Technical Paper, 2004.

[3] R. P. Marimuthu, B.-C. Jang, and S. J. Hong, "A study on SUV parameters sensitivity on rollover propensity," SAE Technical Paper, 2006.

[4] M. A. Hassan, M. A. A. Abdelkareem, G. Tan, and M. M. Moheyeldein, "Conflict and sensitivity analysis of vehicular stability using a two-state linear bicycle model," Shock and Vibration, vol. 2021, Article ID 6641972, 17 pages, 2021.

[5] M. A. Hassan, M. M. Moheyeldein, A. M. M. Abdelkareem, A. Elagouz, and G. Tan, "Advanced study of tire characteristics and their influence on vehicle lateral stability and untripped rollover threshold," Alexandria Engineering Journal, vol. 59, no. 3, pp. 1613-1628, 2020.

[6] T. Shim and P. C. Velusamy, "Influence of suspension properties on vehicle roll stability," SAE Technical Paper, 2006.

[7] G.-M. Dong, N. Zhang, and H.-P. Du, "Investigation into untripped rollover of light vehicles in the modified fishhook and the sine manoeuvres, part II: effects of vehicle inertia property, suspension and tyre characteristics," Vehicle Ssystem Ddynamics, vol. 49, no. 6, pp. 949-968, 2011.

[8] M. A. Abdelkareem, M. M. Makrahy, A. M. Abd-El-Tawwab, A. El-Razaz, M. K. Ahmed Ali, and M. M. Moheyeldein, “An analytical study of the performance indices of articulated truck semi-trailer during three different cases to improve the driver comfort," Proceedings of the Institution of Mechanical Engineers-Part K: Journal of Multi-Body Dynamics, vol. 232, no. 1, pp. 84-102, 2018.

[9] B. Jang and R. Marimuthu, "Sensitivity analysis of SUV parameters on rollover propensity," International Journal of Automotive Technology, vol. 7, no. 6, pp. 703-714, 2006.

[10] M. El-Gindy and W. Kenis, Influence of a Trailer's Axle Arrangement and Loads on the Stability and Control of a Tractor/ Semitrailer, Federal Highway Administration. Office of Engineering, McLean, VA, USA, 1998. 
[11] L. O. García, F. R. Wilson, and J. D. Innes, "Heavy truck dynamic rollover: eEffect of load distribution, cargo type, and road design characteristics," Transportation Rresearch Rrecord, vol. 1851, no. 1, pp. 25-31, 2003.

[12] N. Zhang, G.-M. Dong, and H.-P. Du, "Investigation into untripped rollover of light vehicles in the modified fishhook and the sine maneuvers. Part I: vehicle modelling, roll and yaw instability," Vehicle System Dynamics, vol. 46, no. 4, pp. 271-293, 2008.

[13] A. Hac, Rollover Stability Index Including Effects of Suspension Design, SAE Technical Paper, SAE 2002 World Congress \& Exhibition, Detroit, MI, USA, 2002.

[14] A. Hac, "Influence of chassis characteristics on sustained roll, heave and yaw oscillations in dynamic rollover testing," SAE Technical Paper, 2005.

[15] T. Shim and P. C. Velusamy, "Improvement of vehicle roll stability by varying suspension properties," Vehicle System Dynamics, vol. 49, no. 1-2, pp. 129-152, 2011.

[16] L. Wang, N. Zhang, and H. Du, "Experimental suspension in vehicle dynamics and stability control investigation of a hydraulically interconnected," SAE International Journal of Passenger Cars-Mechanical Systems, vol. 5, no. 2012, pp. 759-768, 2012.

[17] H. Qi, B. Zhang, N. Zhang, M. Zheng, and Y. Chen, "Enhanced lateral and roll stability study for a two-axle bus via hydraulically interconnected suspension tuning," SAE International Journal of Vehicle Dynamics, Stability, and NVH, vol. 3, 2018.

[18] D. Cao, S. Rakheja, and C.-Y. Su, "Dynamic analyses of roll plane interconnected hydro-pneumatic suspension systems," International Jjournal of Vvehicle Ddesign, vol. 47, no. 1-4, pp. 51-80, 2008.

[19] W. A. Smith, N. Zhang, and W. Hu, "Hydraulically interconnected vehicle suspension: handling performance," Vehicle System Dynamics, vol. 49, no. 1-2, pp. 87-106, 2011.

[20] J. Zou, X. Guo, L. Xu et al., "Simulation research of a hydraulic interconnected suspension based on a hydraulic energy regenerative shock absorber," in Proceedings of the WCX World Congress Experience, SAE Technical Paper, Detroit, MI, USA, April 2018.

[21] J. Mi, L. Xu, S. Guo, M. A. A. Abdelkareem, L. Meng, and L. Zuo, "The dimension match and parameters setting of the hydraulic motor for the hydraulic-electromagnetic energyregenerative shock absorber," in Proceedings of the ASME 2017 International Design Engineering Technical Conferences and Computers and Information in Engineering Conference, Cleveland, OH, USA, August 2017.

[22] S. K. Sharma and A. Kumar, "Disturbance rejection and forcetracking controller of nonlinear lateral vibrations in passenger rail vehicle using magnetorheological fluid damper," Journal of Intelligent Material Systems and Structures, vol. 29, no. 2, pp. 279-297, 2018.

[23] S. K. Sharma, "Multibody analysis of longitudinal train dynamics on the passenger ride performance due to brake application," Proceedings of the Institution of Mechanical Engineers-Part K: Journal of Multi-Bbody Dynamics, vol. 233, no. 2, pp. 266-279, 2019.

[24] R. S. Sharp, "Fundamentals of the lateral dynamics of road vehicles," in Mechanics for a New Mellennium, pp. 127-146, Springer, Boston, MA, USA, 2001.

[25] R. Rajamani, "Lateral vehicle dynamics," in Vehicle Dynamics and Ccontrol, pp. 15-46, Springer, Berlin, Germany, 2012.

[26] J. Zou, X. Guo, M. A. A. Abdelkareem, L. Xu, and J. Zhang, "Modeling and ride analysis of a hydraulic interconnected suspension based on the hydraulic energy regenerative shock absorbers," Mechanical Systems and Signal Processing, vol. 127, pp. 345-369, 2019.

[27] M. A. Abdelkareem, M. M. Kaldas, M. K. A. Ali, and L. Xu, "Analysis of the energy harvesting potential-based suspension for truck semi-trailer," Proceedings of the Institution of Mechanical Engineers-Part D: Journal of Automobile Engineering, vol. 233, no. 11, pp. 2955-2969, 2019.

[28] B. Zheng, X. Huang, W. Zhang, R. Zhao, and S. Zhu, “Adhesion characteristics of tire-asphalt pavement interface based on a proposed tire hydroplaning model," Advances in Materials Science and Engineering, vol. 2018, Article ID 5916180, 12 pages, 2018

[29] R. W. Allen, T. T. Myers, T. J. Rosenthal, and D. H. Klyde, "The effect of tire characteristics on vehicle handling and stability," SAE Technical Paper, 2000.

[30] N. D. Smith, Understanding Parameters Influencing Tire Modeling, Department of Mechanical Engineering, Formula SAE Platform, Colorado State University, Fort Collins, CO, USA, 2004.

[31] C.-S. Liu, "Adhesion coefficient of automobile tire and road surface," Journal of Central South University of Technology, vol. 15, no. 1, pp. 210-214, 2008.

[32] Y. Oh and H. Lee, "Characteristics of a tire friction and performances of a braking in a high speed driving," Advances in Mechanical Engineering, vol. 6, Article ID 260428, 2014.

[33] Y.-Q. Zhao, H. Q. Li, F. Lin, J. Wang, and X.-W. Ji, "Estimation of road friction coefficient in different road conditions based on vehicle braking dynamics," Chinese Journal of Mechanical Engineering, vol. 30, no. 4, pp. 982-990, 2017.

[34] B. N. Persson, U. Tartaglino, O. Albohr, and E. Tosatti, "Rubber friction on wet and dry road surfaces: the sealing effect," Physical ReviewB, vol. 71, no. 3, Article ID 035428, 2005.

[35] T. J. Wielenga, "Tire properties affecting vehicle rollover," SAE Technical Paper, 1999.

[36] Z.-F. Wang, M. M. Dong, L. Gu, J. J. Rath, Y. C. Qin, and B. Bai, "Influence of road excitation and steering wheel input on vehicle system dynamic responses," Applied Sciences, vol. 7, no. 6, p. 570, 2017.

[37] Y. Li, W. Sun, J. Huang, L. Zheng, and Y. Wang, "Effect of vertical and lateral coupling between tyre and road on vehicle rollover," Vehicle System Dynamics, vol. 51, no. 8, pp. 1216-1241, 2013.

[38] F. W. Baumann and L. Eckstein, "Effects causing untripped rollover of light passenger vehicles in evasive maneuvers," in Proceeding of the SAE 2004 World Congress \& Exhibition, SAE Technical Paper, Detroit, MI, USA, March 2004.

[39] D. Joshi, S. Kedia, and S. Muthiah, A Study on the Effect of Steering Input Frequency on Transient Lateral Dynamics of Four-Wheeled Passenger Vehicles, SAE Technical Paper, Warrendale, PA, USA, 2019.

[40] H. Taghavifar and S. Rakheja, "Parametric analysis of the potential of energy harvesting from commercial vehicle suspension system," Proceedings of the Institution of $\mathrm{Me}$ chanical Engineers-Part D: Journal of Automobile Engineering, vol. 233, no. 11, pp. 2687-2700, 2019.

[41] R. Zhang, X. Wang, E. A. Shami, S. John, L. Zuo, and C. H. Wang, "A novel indirect-drive regenerative shock absorber for energy harvesting and comparison with a conventional direct-drive regenerative shock absorber," Applied Energy, vol. 229, pp. 111-127, 2018.

[42] R. Zhang, X. Wang, and Z. Liu, "A novel regenerative shock absorber with a speed doubling mechanism and its Monte 
Carlo simulation," Journal of Sound and Vibration, vol. 417, pp. 260-276, 2018.

[43] M. A. A. Abdelkareem, A. B. M. Eldaly, M. K. A. Ali et al., "Monte Carlo sensitivity analysis of vehicle suspension energy harvesting in frequency domain," Journal of Advanced Research, vol. 24, pp. 53-67, 2020.

[44] M. Guiggiani, "Vehicle Model for Handling and Performance," in The Science of Vehicle Dynamics: Handling, Braking, and Ride of Road and Race CarsSpringer, Dordrecht, The Netherlands, 2018.

[45] E. Bakker, H. B. Pacejka, and L. Lidner, "A new tire model with an application in vehicle dynamics studies,"SAE Technical Paper, 2006.

[46] R. P. Marimuthu, B.-C. Jang, and S. J. Hong, "A study on SUV parameters sensitivity on rollover propensity," p. 795, SAE paper, 2006.

[47] M. Abe, Vehicle Handling Dynamics: Theory and Application, Butterworth-Heinemann, Oxford, UK, 2nd edition, 2015. 